\title{
U.S. Hydropower Resource Assessment for
} Ohio

Prepared by: Alison M. Conner

James E. Franctort

Project Manager:

Ben N. Rinehart

Published December 1997

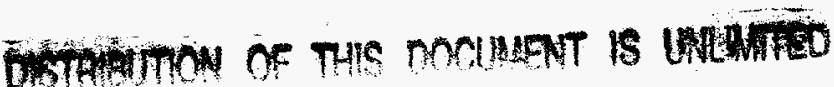

Idaho National Engineering and Environmental Laboratory Renewable Energy Products Department

Lockheed Martin Idaho Technologies Company Idaho Falls, Idaho $\mathbf{8 3 4 1 5}$

Prepared for the

U.S. Department of Energy

Assistant Secretary for Energy Efficiency and Renewable Energy

Under DOE Idaho Operations Office

Contract DE-AC07-94ID13223 


\section{DISCLAIMER}

This report was prepared as an account of work sponsored by an agency of the United States Government. Neither the United States Government nor any agency thereof, nor any of their employees, makes any warranty, express or implied, or assumes any legal liability or responsibility for the accuracy, completeness, or usefulness of any information, apparatus, product, or process disclosed, or represents that its use would not infringe privately owned rights. Reference herein to any specific commercial product, process, or service by trade name, trademark, manufacturer, or otherwise does not necessarily constitute or imply its endorsement, recommendation, or favoring by the United States Government or any agency thereof. The views and opinions of authors expressed herein do not necessarily state or reflect those of the United States Government or any agency thereof. 


\section{DISCLAIMER}

Portions of this document may be illegible electronic image products. Images are produced from the best available original document. 


\begin{abstract}
The U.S. Department of Energy is developing an estimate of the undeveloped hydropower potential in the United States. The Hydropower Evaluation Software (HES) is a computer model that was developed by the Idaho National Engineering Laboratory ${ }^{\mathrm{a}}$ for this purpose. HES measures the undeveloped hydropower resources available in the United States, using uniform criteria for measurement. The software was developed and tested using hydropower information and data provided by the Southwestern Power Administration. It is a menu-driven program that allows the personal computer user to assign environmental attributes to potential hydropower sites, calculate development suitability factors for each site based on the environmental attributes present, and generate reports based on these suitability factors. This report describes the resource assessment results for the State of Ohio.
\end{abstract}

a. In January 1997, the name of the Idaho National Engineering Laboratory (INEL) was changed to the Idaho National Engineering and Environmental Laboratory (INEEL). INEEL will be used throughout the text of the document, except where the use of INEL is historically important. 


\section{ACKNOWLEDGMENTS}

The authors thank Peggy A. M. Brookshier and John V. Flynn of the U.S. Department of Energy for their active participation and timely comments. 


\section{CONTENTS}

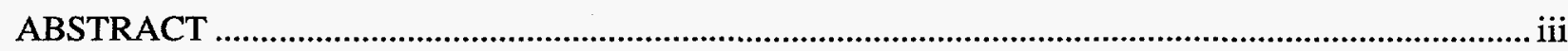

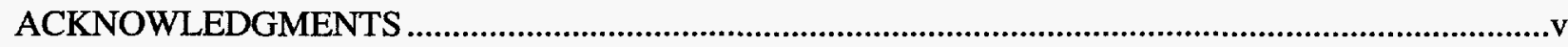

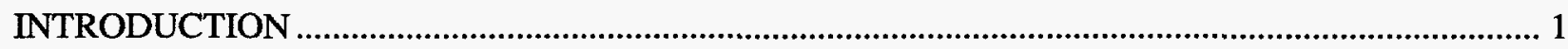

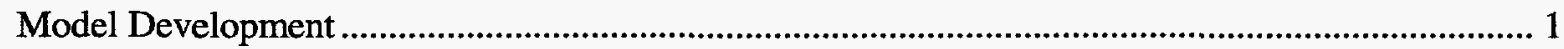

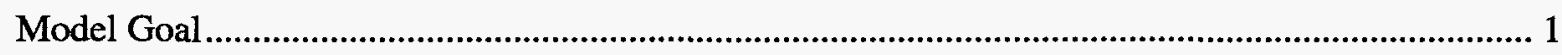

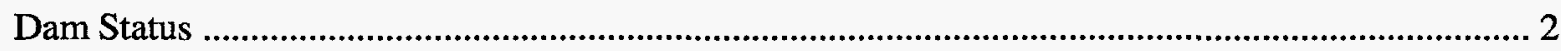

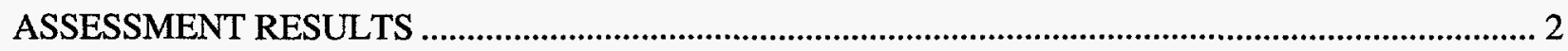

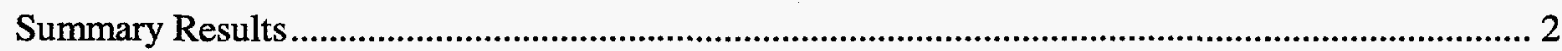

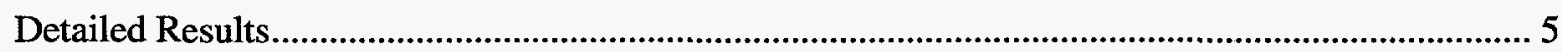

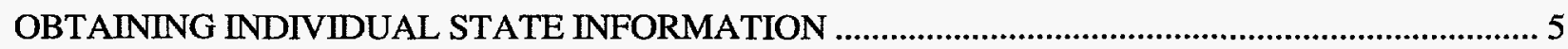

ADDITIONAL HYDROPOWER EVALUATION SOFTWARE INFORMATION .............................. 6

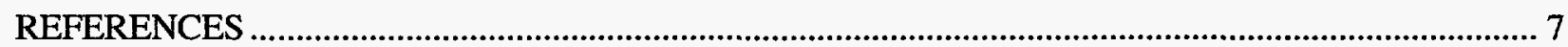

Appendix A-Summary Report

Appendix B-River Basins Report

Appendix C-Ohio Sites List

Appendix D-Individual Resource Database List

\section{FIGURES}

1. Number of sites, by capacity groups, with HES-modeled undeveloped hydropower potential.......... 3

2. The nonmodeled and HES-modeled undeveloped hydropower potential ...................................... 3

3. The number of sites with undeveloped hydropower potential and the total megawatts of HES-modeled undeveloped hydropower potential. 
4. Number of sites with undeveloped hydropower potential in the Ohio river basins

5. Megawatts of HES-modeled undeveloped hydropower potential in the Ohio river basins . 5

\section{TABLES}

1. Undeveloped hydropower potential summary for Ohio 2 


\section{U.S. Hydropower Resource Assessment for Ohio}

\section{INTRODUCTION}

In June 1989, the U.S. Department of Energy initiated the development of a National Energy Strategy to identify the energy resources available to support the expanding demand for energy in the United States. Public hearings conducted as part of the strategy development process indicated that undeveloped hydropower resources were not well defined. As a result, the Department of Energy established an interagency Hydropower Resource Assessment Team to ascertain the undeveloped hydropower potential. In connection with these efforts by the Department of Energy, the Idaho National Engineering Laboratory designed the Hydropower Evaluation Software (HES), which has been used to perform a resource assessment of the undeveloped conventional hydropower potential in over 40 states. This report presents the results of the hydropower resource assessment for the State of Ohio. Undeveloped pumped storage hydropower potential is not included.

The HES was developed as a tool to measure undeveloped hydropower potential regionally or by state. The software is not intended to provide precise development factors for individual sites, but to provide regional or state totals. Because the software was developed as a generic measurement tool encompassing national issues, regional and state totals must be considered judiciously; various local issues may skew undeveloped hydropower potential totals. The information for the resource assessment was compiled from the Federal Energy Regulatory Commission's Hydroelectric Power Resources Assessment database and several other sources. Refer to DOE/ID-10338, the User's Manual (Francfort, Matthews, Rinehart 1991) for the specifics of the software and to DOE/ID-10430.1, the Status Report (Conner, Francfort, Rinehart 1996) for an overview of all resource assessment activities to date.

\section{Model Development}

Hydropower Evaluation Software, both a probability-factor computer model and a database, is a menu-driven program that is intended to be user-friendly. Computer screens and report-generation capabilities were developed to meet the needs of users nationwide. The software uses environmental attribute data to generate an overall project environmental suitability factor (PESF) between 0.1 and 0.9 , where 0.9 indicates the highest likelihood of development and 0.1 indicates the lowest likelihood of development. The suitability factors depend on the unique environmental attributes of each potential site. They reflect the considerations that (a) environmental concerns can make a potential site unacceptable, prohibiting its development (for a suitability factor of 0.1 ), or (b) if there are no environmental concerns, there is no negative effect on the likelihood of site development (for a suitability factor of 0.9). A combination of attributes can result in a lower suitability factor because multiple environmental considerations would reduce the likelihood that a site may be developed to its physical potential.

\section{Model Goal}

The goal of the HES is to assemble an accurate resource database of all sites with undeveloped hydropower potential in the United States for use as a planning tool to determine the viable national hydropower potential. Undeveloped hydropower potential is not limited to the development of new sites; it also includes the development of additional hydropowergenerating capacity at sites that currently have hydropower, but are not developed to their full potential. This undeveloped hydropower potential is a source of nonpolluting, renewable energy available to meet the growing power needs of the United States. The HES should help make this goal obtainable and ensure a set of uniform criteria for national assessment. 


\section{Dam Status}

The effects of environmental attributes vary by dam status. The dam status classifications used are as follows:

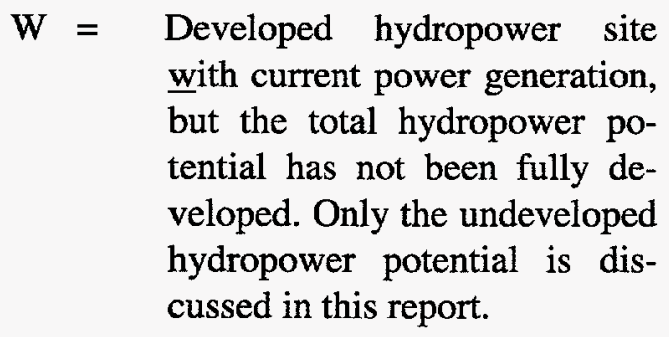

$\mathrm{W} / \mathrm{O}=$ Developed site without current power generation. The site has some type of developed impoundment or diversion structure, but no developed hydropower generating capability.

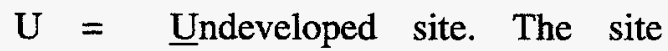
does not have power generation capability nor a developed impoundment or diversion structure.

\section{ASSESSMENT RESULTS Summary Results}

A total of 43 sites (Table 1) have been identified and assessed for their undeveloped hydropower potential. The HES results for individual site capacities range from 25 kilowatts (kW) to 40 megawatts (MW). Greater than one-half $(51 \%)$ of the sites in Ohio are small hydropower sites, less than 1 MW (Figure 1).

The nonmodeled undeveloped hydropower potential total for Ohio was identified as 242 MW. The HES results lowers this estimate about $24 \%$ to $183 \mathrm{MW}$. The greatest reduction in undeveloped hydropower potential, by $\mathrm{MW}$, occurs at sites that have some type of developed impoundment or diversion structure, but no hydropower generating capability (W/O category). These sites have a HES-modeled undeveloped hydropower potential of about 138 $\mathrm{MW}$, a 45-MW reduction in the estimated undeveloped hydropower potential (Figure 2). Figure 3 correlates the number of sites that have undeveloped hydropower potential with the total megawatts of HES-modeled undeveloped hydropower potential.

The 43 identified sites are located within four major river basins and several minor river basins. The number of sites per major river basin ranges from 1 in the Maumee River basin to 29 in the Ohio Main Stream River basin (Figure 4). Ninety-two percent of the undeveloped hydropower potential in the State of Ohio is contained within the Ohio Main Stream River basin (Figure 5).

Table 1. Undeveloped hydropower potential summary for Ohio. The table contains the nonmodeled undeveloped nameplate potential and the HES-modeled undeveloped potential totals.

\begin{tabular}{lccc}
\hline & Number of projects & Nameplate potential & HES-modeled potential \\
& & $($ MW) & $($ MW) \\
\cline { 2 - 4 } With Power & 1 & 2.0 & 1.0 \\
W/O Power & 33 & 183.1 & 138.0 \\
Undeveloped & 9 & 57.0 & 44.2 \\
State Total & 43 & 242.1 & 183.2 \\
\hline
\end{tabular}




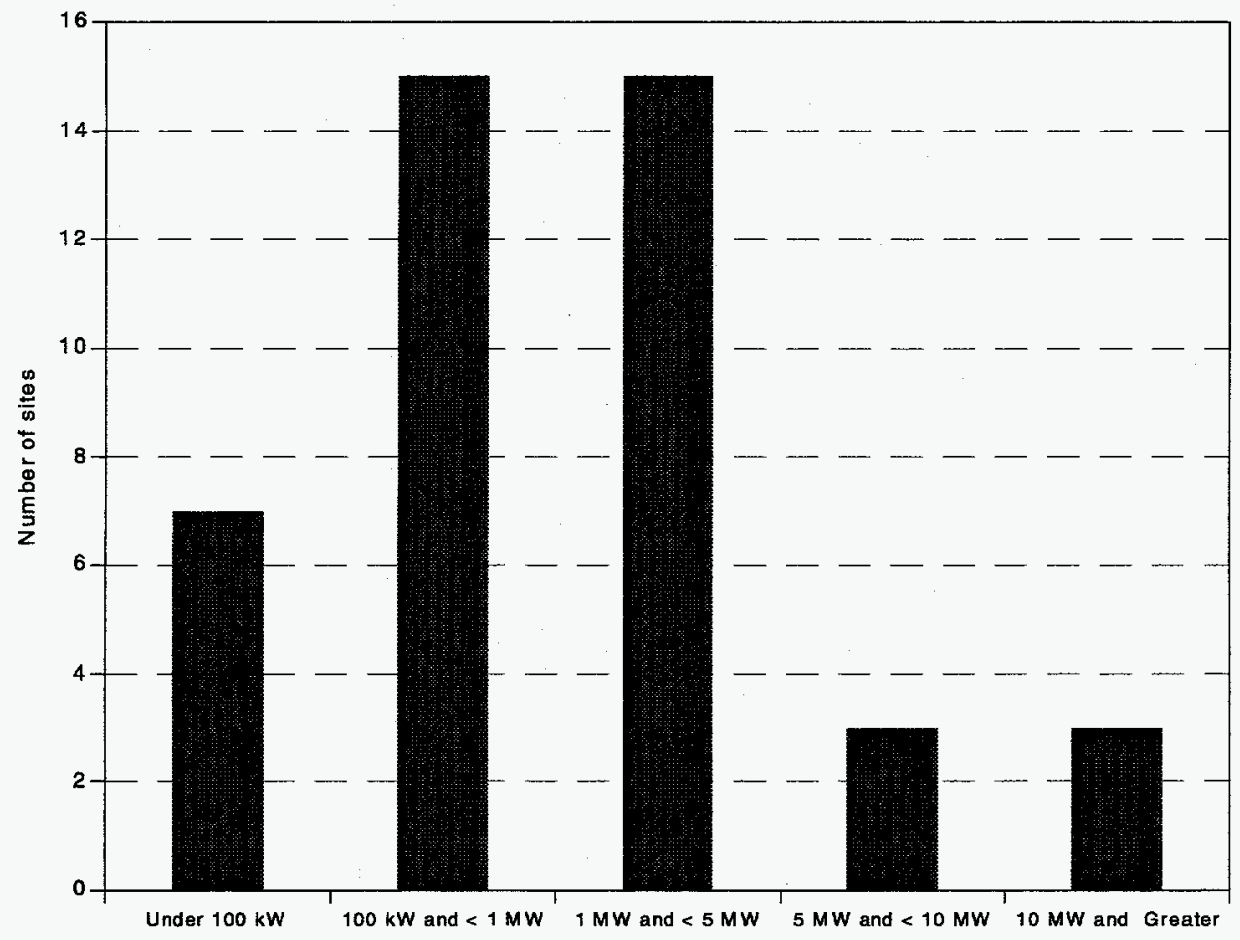

Figure 1. Number of sites, by capacity groups, with HES-modeled undeveloped hydropower potential.

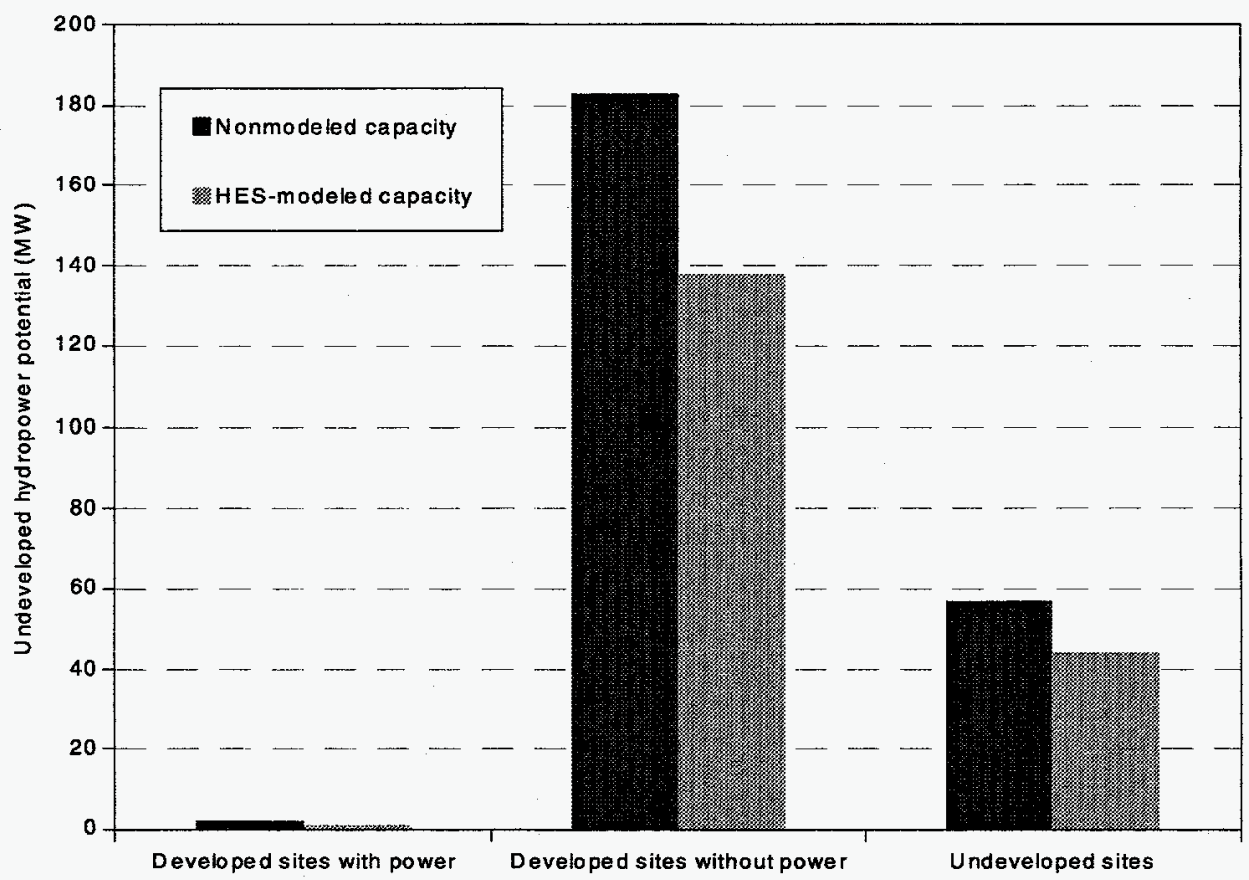

Figure 2. The nonmodeled and HES-modeled undeveloped hydropower potential. 


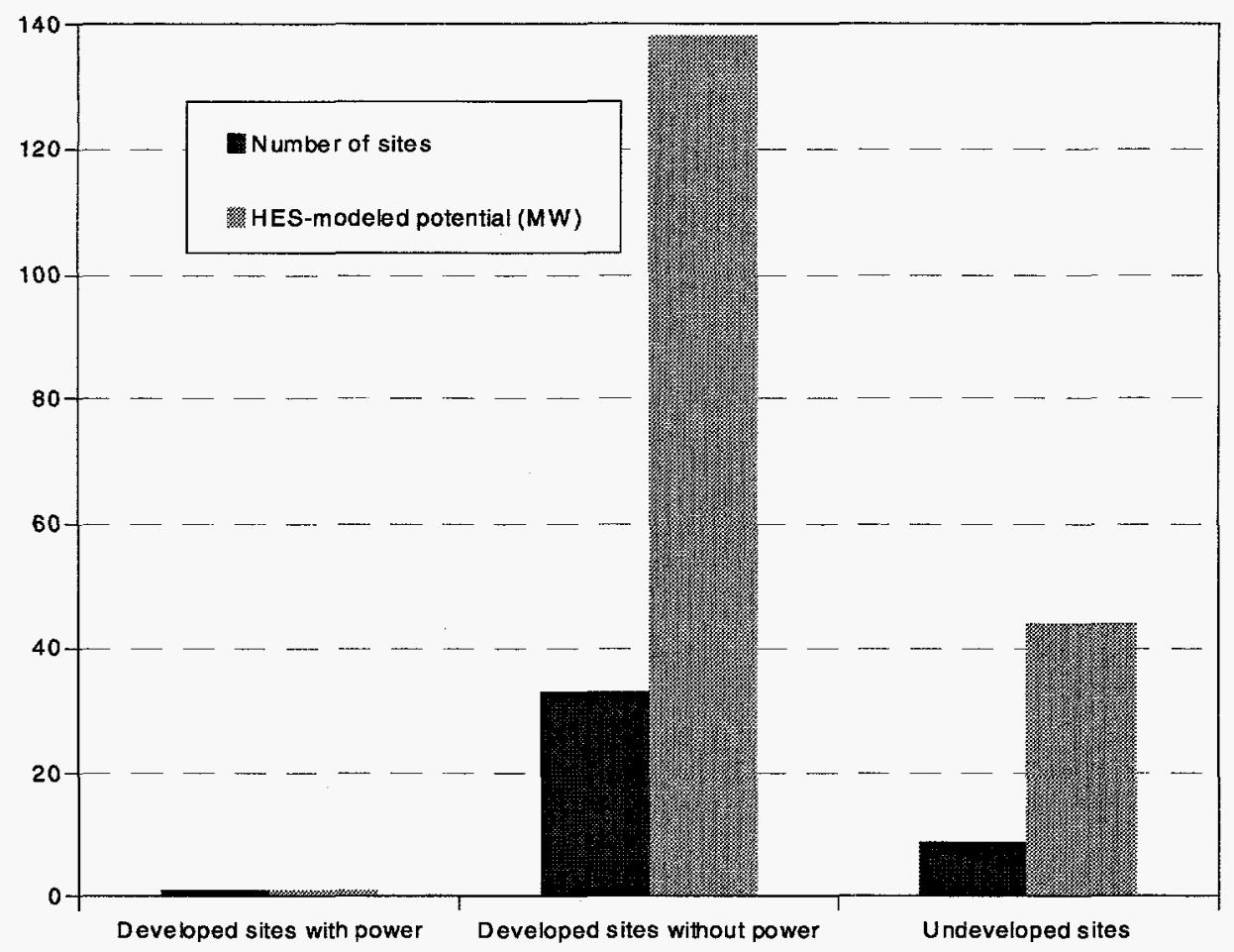

Figure 3. The number of sites with undeveloped hydropower potential and the total megawatts of HES-modeled undeveloped hydropower potential.

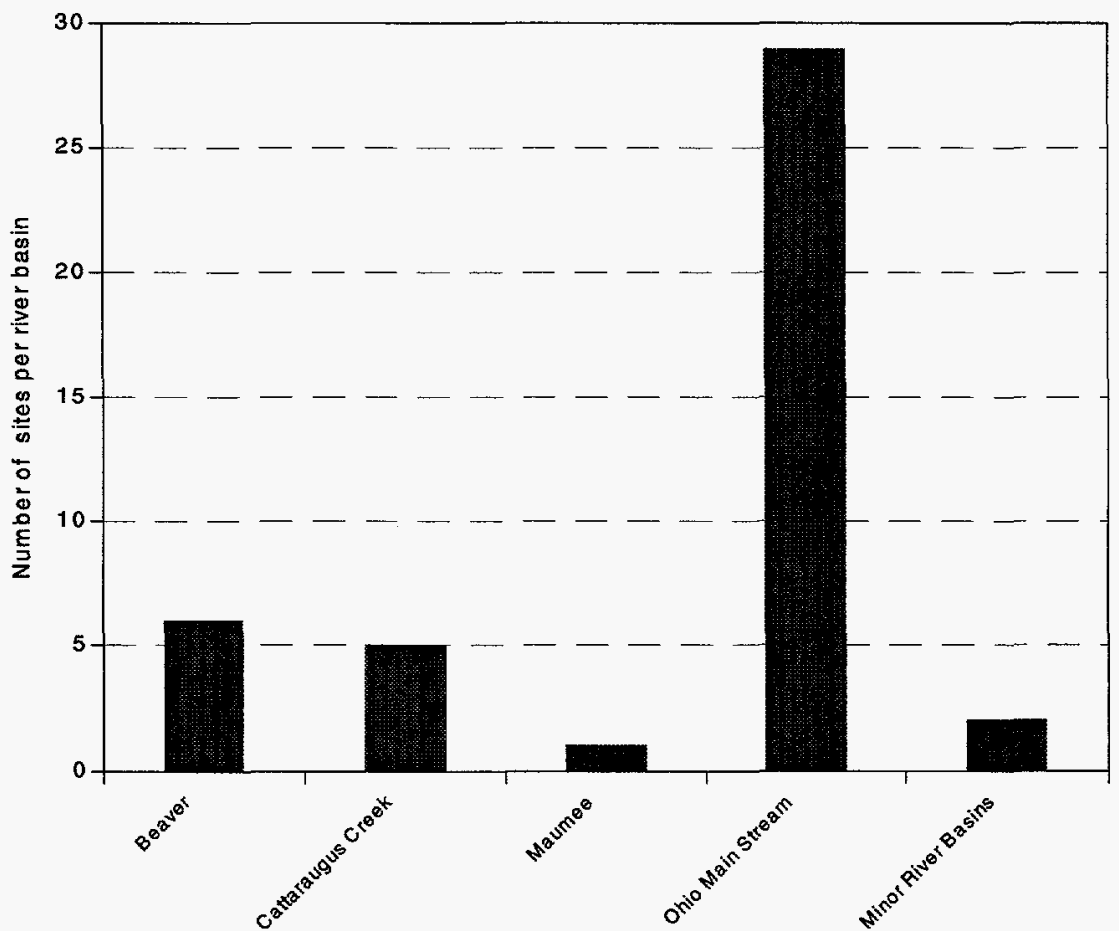

Figure 4. Number of sites with undeveloped hydropower potential in the Ohio river basins. 


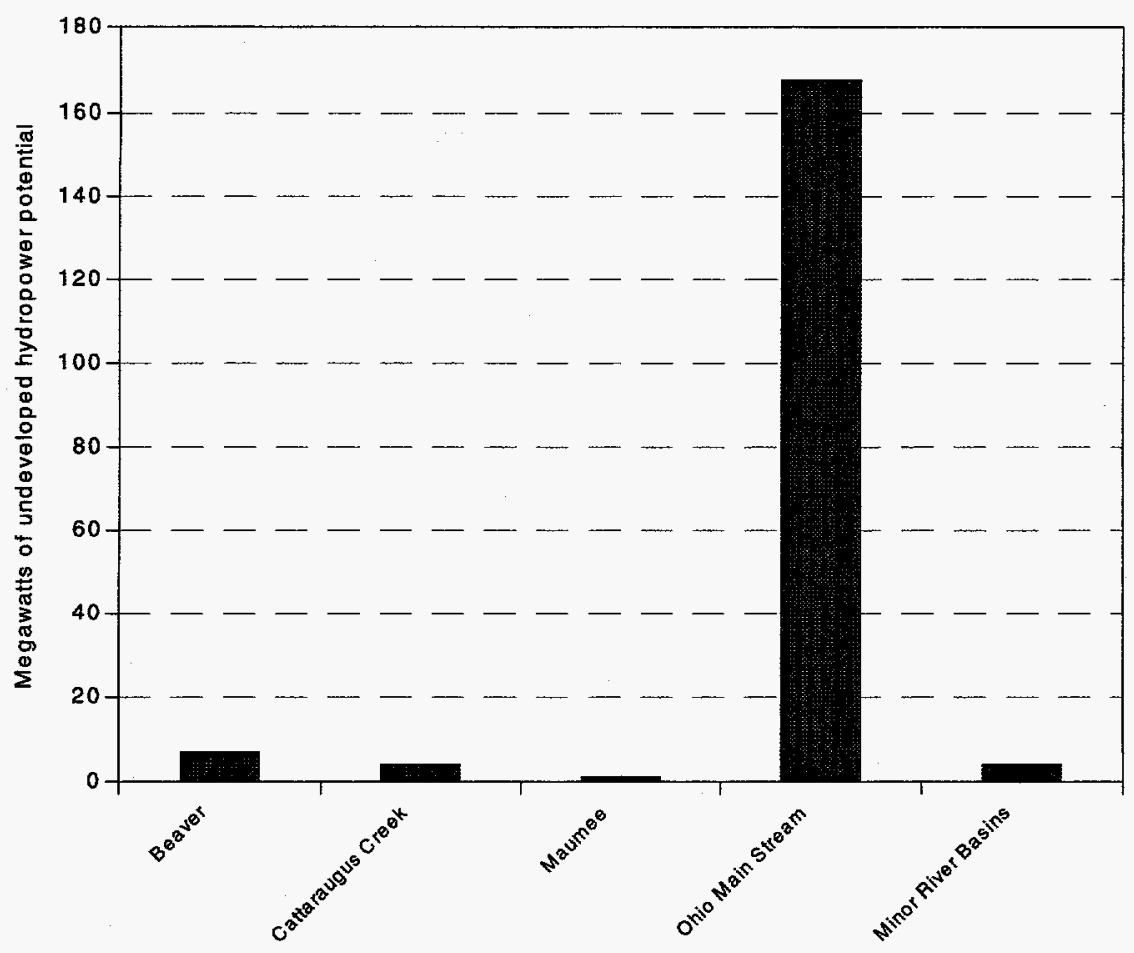

Figure 5. Megawatts of HES-modeled undeveloped hydropower potential in the Ohio river basins.

\section{Detailed Results}

The appendices contain, in the form of HES-generated reports, detailed information about the undeveloped hydropower potential in Ohio. The appendices contain the following information:

Appendix A summarizes the undeveloped hydropower potential by dam status groups. The number of sites, nonmodeled undeveloped hydropower potential, and HES-modeled undeveloped hydropower potential is provided based on the dam status.

Appendix B provides the hydropower resource assessment by river basin, which includes the project number, project name, stream name, dam status, nonmodeled undeveloped hydropower potential, and the HES-modeled undeveloped hydropower potential for each site. Subtotals are provided for each river basin.

Appendix C lists the project numbers, plant name, stream name, if a site is Federally owned, nonmodeled undeveloped hydropower potential, and HES-modeled undeveloped hydropower potential. The sites are grouped by dam status.

Appendix D contains a resource database list for the 43 sites in Ohio. Information includes plant name, stream, state, county, river basin and owner names, project number, nameplate and HES-modeled undeveloped hydropower potential, the unit and plant types, dam status, latitude, longitude, and the environmental factors that the HES uses to determine the PESF.

\section{OBTAINING INDIVIDUAL STATE INFORMATION}

Additional copies of the hydropower resource assessment results for individual states are available and can be obtained by writing or calling the authors or the National Technical Information Service (NTIS).

Telephone Orders-(703) 487-4650. NTIS sales desk and customer services are available between 8:30 a.m. and 5:00 p.m., Eastern Standard Time. 
Fax-(703) 321-8547. Customers may fax their orders to NTIS. These orders may be charged to a NTIS deposit account, American Express, VISA, or MasterCard.

Mail Orders-Mail orders should be sent to National Technical Information Service, Document Sales, 5285 Port Royal Road, Springfield, VA 22161. Call the sales desk for prices before placing an order.

E-mail-Customers may e-mail their requests to info@ntis.fedworld.gov.

Method of Payment-Customers may pay for reports (and other NTIS products and services) by (a) credit card (American Express, Visa or MasterCard); (b) check or money order on a United States bank payable to NTIS; (c) NTIS deposit account; or, (d) by asking to be billed (add $\$ 7.50$ per order), United States, Canada, and Mexico, only.

Handling Fee-A $\$ 4.00$ handling fee per total order applies to orders from the United States, Canada, and Mexico. Handling charges do not apply to rush order service or pick-up orders.

Postage and Shipping-Orders are shipped first class mail, or equivalent, to addresses in the United States, Canada, and Mexico.

Order Turnaround Time-Orders for technical reports generally are shipped within 3 to 5 days of receipt. For faster service, NTIS offers rush order service.

Rush Order Service-Call 1-800-533-NTIS. In Ohio, Canada, and Mexico call (703) 487-4700.
For NTIS rush order service add $\$ 15$ per item. This guarantees that an order will be processed through NTIS within 24 hours of its receipt. These orders receive immediate, individual attention. The items ordered are delivered by first call mail. Call NTIS for information on rush order service for computer products.

For Help in Tracing an Order-Call (703) 487-4650 and request the customer service option.

\section{ADDITIONAL HYDROPOWER EVALUATION SOFTWARE INFORMATION}

Additional information concerning the HES can be obtained by contacting Ben Rinehart or Jim Francfort at the addresses provided below. Copies of the software and the User's Manual may also be obtained from these individuals.

Ben Rinehart, Project Manager

Idaho National Engineering and Environmental Laboratory

P.O. Box 1625, MS 3830

Idaho Falls, ID 83415-3830

(208) 526-1002

Jim Francfort

Idaho National Engineering and Environmental Laboratory

P.O. Box 1625, MS 3830

Idaho Falls, ID 83415-3830

(208) 526-6787 


\section{REFERENCES}

Conner, A. M., J. E. Francfort, and B. N. Rinehart, 1996, Uniform Criteria for U.S. Hydropower Resource Assessment, Hydropower Evaluation Software Status Report-II, DOE/ID 10430.1, Idaho National Engineering Laboratory, Idaho Falls, Idaho.

Francfort, J. E., S. D. Matthews, and B. N. Rinehart, 1991, Hydropower Evaluation Software User's Manual, DOE/ID-10338, Idaho National Engineering Laboratory, Idaho Falls, Idaho. 
Appendix A Summary Report 
DATE: $12 / 01 / 97$

H Y D R O P O W E R

C A P A C I T Y

S U M M A R Y

State

Category

Number of

Projects

TOTALS

GRAND TOTAL
With Power

w/O Power

Undeveloped

with Power 1

W/O Power

Undeveloped

STATE TOTAL

33

9

43

1

33

9

43

1
Name Plate

Capacity (KW)

1950

183120

56990

242060

1950

183120

56990

242060
HES Adjusted Capacity (KW)
137975.50

44235

183185.50

975

137975.50

44235

183185.50 
Appendix B

River Basins Report 
Page No.

$12 / 01 / 97$

FERC

Number

$$
\text { Plant Name/ }
$$

stream

$\begin{array}{ll}\text { ** River Basin BEAVER RIVER BASIN } \\ \text { OH010 } & \begin{array}{l}\text { LAKE MILTON } \\ \text { MAHONING R }\end{array} \\ 10252 & \begin{array}{l}\text { BOOSTER STA } \\ \text { OWSC PL(MAHONING R) }\end{array} \\ \text { OH008 } & \begin{array}{l}\text { MOSQUITO CREEK } \\ \text { MOSQUITO CR, MAHONING R }\end{array} \\ \text { OH009 } & \begin{array}{l}\text { MICHAEL J KIRWIN } \\ \text { W BR MAHONING R }\end{array} \\ 09972 & \begin{array}{l}\text { MILTON } \\ \text { MAHONING R }\end{array} \\ 03359 & \begin{array}{l}\text { BERLIN IAKE } \\ \text { MAHONING R }\end{array} \\ \text { ** Subtotal ** }\end{array}$

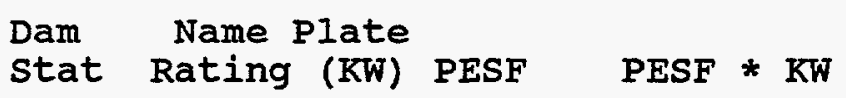

$\mathrm{U}$

$2570.00 \quad 0.90$

2313.00

U

$200.00 \quad 0.90$

180.00

wo

$1100.00 \quad 0.90$

990.00

wo

$1470.00 \quad 0.90$

1323.00

wo

$1000.00 \quad 0.90$

900.00

wo

$1560.00 \quad 0.75$

1170.00

7900.00

6876.00

** River Basin CATTARAUGUS CREEK BASIN

03279 KENT DAM

$\mathrm{U}$

$$
420.00 \quad 0.10
$$

42.00

OHOO1 FREMONT

$\mathrm{U}$

$4700.00 \quad 0.10$

470.00

SANDUSKY $R$

10619

CUYAHOGO FALLS

CUYAHOGA $R$

wo

$5500.00 \quad 0.50$

2750.00

03088

ROCKWELI

CUYAHOGA $R$

wo

$680.00 \quad 0.50$

340.00

wo

$650.00 \quad 0.50$

325.00

EAST BR, BLACK R

11950.00

3927.00

W

$1950.00 \quad 0.50$

975.00

AUGLAIZE $R$

** River Basin MAUMEE RIVER BASIN

06637 DEFIANCE (AUGLAIZE R)

** Subtotal ** 
Page No.

$12 / 01 / 97$

FERC

Number

** River Basin MINOR RIVER BASINS

10860 EAST FORK

E FK LITTLE MIAMI R

07933 CAESAR CREEK

CAESAR CR, LITTLE MIAMI $R$

** Subtotal **

** River Basin OHIO MAIN STREAM

OHOO5

PIQUA

MIAMI $R$

06079 DEEP CREEK

DEEP CR, SCIOTO $R$

06075 ALUM CREEK

ALUM CR, SCIOTO $R$

06074 DELAWARE DAM

OLENTANGY $R$

OHO06 RACINE I\&D
OHIO R

02724 HAMILTON

MIAMI $\mathrm{R}$

OHOO2 DAYTON LOW DAM

GREAT MIAMI $R$

OH003 C J BROWN

BUCK CR, MAD R

OHOO4 ISLAND PARK

GREAT MIAMI $R$

07871 PAINT CREEK

PAINT CR, SCIOTO R

03171 HOOVER

BIG WAINUT CR, SCIOTO $R$

02946 GRIGGS

SCIOTO $\mathrm{R}$

09042 GALLIPOLIS L\&D

OHIO R wo

Dam Name Plate

Stat Rating (KW) PESF PESF * KW

wo

$4000.00 \quad 0.50$

2000.00

7000.00

3500.00

$\mathrm{U}$

$800.00 \quad 0.90$

720.00

$\mathrm{U}$

$2200.00 \quad 0.10$

220.00

U

$900.00 \quad 0.10$

90.00

$\mathrm{U}$

$1200.00 \quad 0.50$

600.00

U

$44000.00 \quad 0.90$

39600.00

wo

$750.00 \quad 0.90$

675.00

wo

$50.00 \quad 0.50$

25.00

wo

$99.00 \quad 0.50$

49.50

wo

$50.00 \quad 0.50$

25.00

wo

$8000.00 \quad 0.50$

4000.00

wo

$500.00 \quad 0.10$

50.00

wo

$2100.00 \quad 0.10$

210.00

wo

$48000.00 \quad 0.75$

36000.00 
Page No.

$12 / 01 / 97$

FERC

Number

3

HYDROPOWER RESOURCE ASSESSMENT BY RIVER BASIN

Plant Name/

stream
Dam Name Plate

Stat Rating (KW) PESF

wo

MUSKINGUM $R$

06998 MUSKINGUM L\&D 3

MUSKINGUM R

07234 BEVERLY L\&D 4

MUSKINGUM $R$

07261 LUKE CHUTE I\&D 5

MUSKINGUM R

08376 MUSKINGUM L\&D 6

MUSKINGUM $\mathbf{R}$

07262 MCCONNELSVILLE L\&D 7

MUSKINGUM $R$

07222 ROKEBY L\&D 8

MUSKINGUM R

07221 PHILO L\&D 9

MUSKINGUM $R$

06563 MUSKINGUM IL\&D 10

MUSKINGUM $R$

07220 ELLIS L\&D 11

MUSKINGUM $\mathbf{R}$

08268 DILLON

LICKING $\mathrm{R}$

08269 WILLS CREEK

WILLS CR, MUSKINGUM $R$

08270 PLEASANT HILL

CLEAR FORK $\mathbf{R}$

03266 PIEDMONT DAM

STILLWATER CR, TUSCARAWAS R

OH007 DOVER

TUSCARAWAS $R$

03218 PIKE ISLAND

OHIO $R$ wo

พo

wo

wo

wo

พo

wo

พอ

wo

พอ

wo

พo

พั

wo

พo

$$
7300.00 \quad 0.90
$$

$10000.00 \quad 0.90$

$5000.00 \quad 0.90$

$4000.00 \quad 0.90$

$1500.00 \quad 0.90$

$4500.00 \quad 0.90$

$5000.00 \quad 0.90$

$5800.00 \quad 0.90$

$930.00 \quad 0.75$

$5000.00 \quad 0.90$

$2100.00 \quad 0.75$

$2400.00 \quad 0.75$

$1160.00 \quad 0.75$

$300.00 \quad 0.75$

$121.00 \quad 0.50$

$49500.00 \quad 0.75$
4050.00

4500.00

6570.00

9000.00

4500.00

3600.00

1350.00

4500.00

5220.00

697.50

1575.00

1800.00

870.00

225.00

60.50

37125.00 
Page No.

$12 / 01 / 97$

FERC

Number

** Subtotal **

*** Total ***

4

HYDROPOWER RESOURCE ASSESSMENT BY RIVER BASIN

Plant Name/

stream
Dam Name Plate

stat Rating (KW) PESF PESF * KW
213260.00

242060.00
167907.50

1831.85 .50 
Appendix C

Ohio Sites List 
Page No.

$12 / 01 / 97$

HYDROPOWER RESOURCE ASSESSMENT BY FERC NUMBER

Plant Name/ stream

** FERC Number 03279

KENT DAM

CUYAHOGA $R$

** Subtotal **

** FERC Number 06074

DELAWARE DAM

OLENTANGY $\mathbf{R}$

** Subtotal **

** FERC Number 06075

ALUM CREEK

ALUM CR, SCIOTO R

** Subtotal **

** FERC Number 06079

DEEP CREEK

DEEP CR, SCIOTO $R$

** Subtotal **

** FERC Number 10252

BOOSTER STA

OWSC PL (MAHONING R)

** Subtotal **

** FERC Number OHOO1

FREMONT

SANDUSKY $R$

** Subtotal **

** FERC Number OH005

PIQUA

MIAMI R

$\begin{array}{lll}\text { Dam } & \text { Name Plate } & \\ \text { ST Stat } & \text { Rating (KW) PESF } & \text { PESF * KW }\end{array}$

$\mathrm{OH} \mathrm{U}$

$420.00 \quad 0.10$

42.00

420.00

42.00

$\mathrm{OH} \quad \mathrm{U}$

$1200.00 \quad 0.50$

600.00

1200.00

600.00

$\mathrm{OH} \mathrm{U}$

$900.00 \quad 0.10$

90.00

900.00

90.00

$\mathrm{OH} \quad \mathrm{U}$

$2200.00 \quad 0.10$

220.00

2200.00

220.00

$\mathrm{OH} \quad \mathrm{U}$

$200.00 \quad 0.90$

180.00

200.00

180.00

$\mathrm{OH} \quad \mathrm{U}$

$4700.00 \quad 0.10$

470.00

4700.00

470.00

$\mathrm{OH} \quad \mathrm{U}$

$800.00 \quad 0.90$

720.00 
HYDROPOWER RESOURCE ASSESSMENT BY FERC NUMBER

\section{Plant Name/}

stream

** Subtotal **

** FERC Number 0 HOO6

RACINE I\&D

OHIO $\mathbf{R}$

** Subtotal **

** FERC Number OH010

LAKE MILTON

MAHONING $\mathbf{R}$

** Subtotal **

** FERC Number 06637

DEFIANCE (AUGLAIZE R)

AUGLAIZE $R$

** Subtotal **

** FERC Number 02724

HAMILTON

MIAMI $\mathbf{R}$

** Subtotal **

** FERC Number 02946

GRIGGS

SCIOTO $\mathrm{R}$

** Subtotal **

** FERC Number 03088

ROCKWELL

CUYAHOGA $R$

** Subtotal **

$\begin{array}{lll}\text { Dam } & \text { Name Plate } & \\ \text { ST Stat } & \text { Rating (KW) PESF } & \text { PESF } * \mathrm{KW}\end{array}$

800.00

720.00

$\mathrm{OH} \mathrm{U}$

$44000.00 \quad 0.90$

39600.00

44000.00

39600.00

$\mathrm{OH} \quad \mathrm{U}$

$2570.00 \quad 0.90$

2313.00

2570.00

2313.00

$\mathrm{OH} \quad \mathrm{W}$

$1950.00 \quad 0.50$

975.00

1950.00

975.00

$\mathrm{OH}$ WO

$750.00 \quad 0.90$

675.00

750.00

675.00

OH Wo

$2100.00 \quad 0.10$

210.00

2100.00

210.00

$\mathrm{OH} \quad$ WO

$680.00 \quad 0.50$

340.00

680.00

340.00 
Page No.

HYDROPOWER RESOURCE ASSESSMENT BY FERC NUMBER

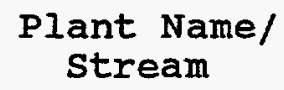

** FERC Number 03171

HOOVER

BIG WALNUT CR,SCIOTO $R$

** Subtotal **

** FERC Number 03218

PIKE ISLAND

OHIO $R$

** Subtotal **

** FERC Number 03266

PIEDMONT DAM

STILLWATER CR, TUSCARAWAS R

** Subtotal **

** FERC Number 03359

BERLIN LAKE

MAHONING $\mathbf{R}$

** Subtotal **

** FERC Number 06563

MUSKINGUM L\&D 10

MUSKINGUM $\mathbf{R}$

** Subtotal **

** FERC Number 06998

MUSKINGUM L\&D 3

MUSKINGUM $R$

** Subtotal **

** FERC Number 07220

ELLIS L\&D 11

MUSKINGUM $\mathrm{R}$
Dam Name Plate
ST Stat Rating (KW) PESF

$\mathrm{OH}$ WO

$$
500.00 \quad 0.10
$$

500.00

$\mathrm{OH}$ WO

$49500.00 \quad 0.75$

49500.00

$\mathrm{OH}$ WO

$300.00 \quad 0.75$

300.00

$\mathrm{OH}$ พO

$1560.00 \quad 0.75$

1560.00

OH Wo

$930.00 \quad 0.75$

930.00

$\mathrm{OH}$ พ०

$10000.00 \quad 0.90$

10000.00

OH wo
$5000.00 \quad 0.90$
9000.00

PESF * KW

50.00

50.00

37125.00

37125.00

225.00

225.00

1170.00

1170.00

697.50

697.50

9000.00

4500.00 
HYDROPOWER RESOURCE ASSESSMENT BY FERC NUMBER

Plant Name/

Stream

** Subtotal **

** FERC Number 07221

PHILO L\&D 9

MUSKINGUM $\mathbf{R}$

** Subtotal **

** FERC Number 07222

ROKEBY L\&D 8

MUSKINGUM $\mathbf{R}$

** Subtotal **

** FERC Number 07234

BEVERLY L\&D 4

MUSKINGUM $R$

** Subtotal **

** FERC Number 07237

ELYRIA

EAST BR, BLACK R

** Subtotal **

** FERC Number 07261

IUKE CHUTE L\&D 5

MUSKINGUM $R$

** Subtotal **

** FERC Number 07262

MCCONNELSVILLE L\&D 7

MUSKINGUM $\mathrm{R}$

** Subtotal ** $\begin{array}{lll}\text { Dam } & \text { Name Plate } \\ \text { ST Stat } & \text { Rating (KW) PESF } \quad \text { PESF * KW }\end{array}$

5000.00

4500.00

OH WO

$5800.00 \quad 0.90$

5220.00

5800.00

5220.00

OH wo

$5000.00 \quad 0.90$

$45,00.00$

5000.00

4500.00

OH Wo

$5000.00 \quad 0.90$

4500.00

5000.00

4500.00

$\mathrm{OH}$ พO

$650.00 \quad 0.50$

325.00

650.00

325.00

OH WO

$4000.00 \quad 0.90$

3600.00

4000.00

3600.00

OH wo

$4500.00 \quad 0.90$

4050.00

4500.00

4050.00 
HYDROPOWER RESOURCE ASSESSMENT BY FERC NUMBER

Plant Name/ Stream

** FERC Number 07751

DEVOLA L\&D 2

MUSKINGUM $R$

** Subtotal **

** FERC Number 07871

PAINT CREEK

PAINT CR, SCIOTO R

** Subtotal **

** FERC Number 07933

CAESAR CREEK

CAESAR CR, LITTLE MIAMI $R$

** Subtotal **

** FERC Number 08268

DILLON

LICKING $R$

** Subtotal **

** FERC Number 08269

WILLS CREEK

WILLS CR, MUSKINGUM $R$

** Subtotal **

** FERC Number 08270

PLEASANT HILL

CLEAR FORK $R$

** Subtotal **

** FERC Number 08376

MUSKINGUM ILD 6

MUSKINGUM $R$

$\begin{array}{lll}\text { Dam } & \text { Name Plate } & \\ \text { ST Stat } & \text { Rating (KW) PESF } & \text { PESF * KW }\end{array}$

$\mathrm{OH}$ wO

$7300.00 \quad 0.90$

6570.00

7300.00

6570.00

OH wo

$8000.00 \quad 0.50$

4000.00

8000.00

4000.00

$\mathrm{OH}$ wo

$3000.00 \quad 0.50$

1500.00

3000.00

1500.00

$\mathrm{OH}$ WO

$2100.00 \quad 0.75$

1575.00

2100.00

1575.00

$\mathrm{OH}$ wo

$2400.00 \quad 0.75$

1800.00

2400.00

1800.00

$\mathrm{OH}$ wo

$1160.00 \quad 0.75$

870.00

1160.00

870.00

$\mathrm{OH}$ WO

$1500.00 \quad 0.90$

1350.00 
HYDROPOWER RESOURCE ASSESSMENT BY FERC NUMBER

\section{Plant Name/} stream

** Subtotal **

** FERC Number 09042

GALLIPOLIS L\&D

OHIO $\mathbf{R}$

** Subtotal **

** FERC Number 09972

MILTON

MAHONING $\mathbf{R}$

** Subtotal **

** FERC Number 10619

CUYAHOGO FALLS

CUYAHOGA $R$

** Subtotal **

** FERC Number 10860

EAST FORK

E FK LITTLE MIAMI $R$

** Subtotal **

** FERC Number $0 \mathrm{HOO2}$

DAYTON LOW DAM

GREAT MIAMI $R$

** Subtotal **

** FERC Number OH003

C J BROWN

BUCK CR,MAD R

** Subtotal ** $\begin{array}{lll}\text { Dam } & \text { Name Plate } & \\ \text { ST Stat } & \text { Rating (KW) PESF } \quad \text { PESF * KW }\end{array}$

$$
1500.00
$$

OH WO

$48000.00 \quad 0.75$

36000.00

48000.00

36000.00

$\mathrm{OH}$ wo

$1000.00 \quad 0.90$

900.00

1000.00

900.00

$\mathrm{OH}$ พо

$5500.00 \quad 0.50$

2750.00

5500.00

2750.00

$\mathrm{OH}$ พо

$4000.00 \quad 0.50$

2000.00

4000.00

2000.00

OH พ०

$50.00 \quad 0.50$

25.00

50.00

25.00

OH wo

$99.00 \quad 0.50$

49.50

99.00

49.50 
Page No.

$12 / 01 / 97$

HYDROPOWER RESOURCE ASSESSMENT BY FERC NUMBER

Plant Name/

Stream

** FERC Number OHOO4

ISLAND PARK

GREAT MIAMI R

** Subtotal **

** FERC Number 0 H0O7

DOVER

TUSCARAWAS $\mathbf{R}$

** Subtotal **

** FERC Number $0 \mathrm{HOO8}$

MOSQUITO CREEK

MOSQUITO CR, MAHONING $R$

** Subtotal **

** FERC Number OHOO9

MICHAEL J KIRWIN

$W$ BR MAHONING $R$

** Subtotal **

*** Total ***
Dam Name Plate

ST Stat Rating (KW) PESF

PESF * KW

$\mathrm{OH}$ WO

$50.00 \quad 0.50$

25.00

50.00

25.00

$\mathrm{OH}$ wo

$121.00 \quad 0.50$

60.50

121.00

60.50

$\mathrm{OH}$ WO

$1100.00 \quad 0.90$

990.00

1100.00

990.00

$\mathrm{OH}$ WO

$1470.00 \quad 0.90$

1323.00

1470.00

1323.00

242060.00

183185.50 
Appendix D

Individual Resource Database List 
DATE: $12 / 01 / 97$

FERC

Number

02724

HAMILTON

County Name

BUTLER
Plant Name

MIAMI R

River Basin

OHIO MAIN STREAM

PAGE NO: 1

State

stream

Name

$\mathrm{OH}$

\section{Class Owner Name}

M HAMILTON, CITY OF

Name Plate

Rating (KW)

Annual Energy

750.00

PESF

Rating (MWh)

PESF Annual Energy Rating (MWh)

0.90675 .00

3500.00

3150

$\begin{array}{cccccr}\text { Unit } & \text { Plant } & \text { Project } & \text { Dam } & \text { Latitude } & 3929.00 \\ \text { Type } & \text { Type } & \text { Status } & \text { Status } & \text { Longitude } & 8430.00 \\ \text { C } & \text { ROR } & \text { No } & \text { wo } & \end{array}$

Factor

Wild/scenic Protection Wild/Scenic Tributary or Upstream/Downstream wild/Scenic Location Cultural Value Fish Presence Value Geologic Value Historic Value other Value Recreation value Scenic Value
Exist Prob

Factor

Exist Prob

0.90

Wildlife Value Threatened/Endangered Fish Threatened/Endangered wildlife

0.90 Federal Land Code 103

0.90 Federal Land Code 104

0.90 Federal Land Code 105

0.90 Federal Land Code 106

0.90 Federal Land Code 107

0.90 Federal Land Code 108

0.90 Federal Land Code 198
0.90

0.90

0.90

0.90

0.90

0.90

0.90

0.90

0.90

0.90 
DATE: $12 / 01 / 97$

FERC

Number

02946

GRIGGS

Plant Name

County Name

FRANKLIN

Class

Owner Name

$\mathbf{M}$

COLUMBUS, CITY OF

Name Plate

Rating (KW)

PESF

$\mathrm{PESF} * \mathrm{KW}$

Annual Energy

Rating (MWh)

6500.00

PAGE NO: 2

stream

State

Name

$\mathrm{OH}$

$$
2100.00
$$

$0.10 \quad 210.00$

Dam
Status

Latitude

4001.00

Unit

Plant
Type
STG

Project status

พั

Factor

Exist Prob

Factor

Exist Prob

Wild/Scenic Protection

Wild/Scenic Tributary or Upstream/ Downstream wild/Scenic Location

Cultural value

Fish Presence Value

Geologic Value

Historic Value

other Value

Recreation Value

Scenic Value

Exist Prob

0.90

Wildlife Value

Threatened/Endangered Fish

Threatened/Endangered Wildlife

0.90

0.90

0.90

0.90

$\mathrm{Y} \quad 0.75$

0.90

$\mathrm{Y} \quad 0.75$

Federal Iand Code 103

Federal Land Code 104

Federal Land Code 105

Federal Land Code 106

Federal Land Code 107

Federal Land Code 108

Federal Land Code 198
PESF Annual

Energy Rating (MWh)

650

0.90

0.90

0.75

0.90

0.10

0.90

0.90

0.90

0.90

0.90

0.90 

R E S O U R C E
D A T A B A S E
I I S T I N G

DATE: $12 / 01 / 97$

PAGE NO: 3

FERC

Number

03088

ROCKWEIL

county Name

PORTAGE

Plant Name

CUYAHOGA $\mathbf{R}$

stream

River Basin

CATTARAUGUS CREEK BASIN
Class Owner Name

M AKRON, CITY OF

Name Plate

Rating (KW)

PESF

Annual Energy

680.00

$0.50 \quad 340.00$

2100.00

Latitude

Longitude

status

Wo

$\begin{array}{cccclc}\text { Unit } & \text { Plant } & \text { Project } & \text { Dam } & \text { Latitude } & 0.00 \\ \text { Type } & \text { Type } & \text { Status } & \text { Status } & \text { Longitude } & 0.00 \\ & \text { ROR } & \text { XX } & \text { Wo } & \end{array}$

state

Name

$\mathrm{OH}$
Factor

wild/Scenic Protection Wild/Scenic Tributary or Upstream/Downstream wild/scenic Iocation Cultural value Fish Presence Value Geologic Value Historic Value other Value Recreation Value scenic Value
Exist Prob

$\mathrm{Y} \quad 0.50$

wildlife Value Threatened/Endangered Fish Threatened/Endangered Wildlife

0.90 Federal Iand code 103

0.90 Federal Land Code 104

Y 0.75 Federal Land Code 105

0.90 Federal Iand code 106

0.90 Federal Land code 107

0.90 Federal Land code 108

$\mathrm{Y} \quad 0.75$ Federal Land code 198

$\mathrm{Y} \quad 0.90$
0.00

PESF Annual Energy Rating (MWh)

1050

Exist Prob

0.90

0.75

0.90

0.90

0.90

0.90

0.90

0.90

0.90

0.90 
DATE: $12 / 01 / 97$

FERC

Number

Plant Name

03171 HOOVER

County Name

FRANKLIN

class

Owner Name

M COLUMBUS, CITY OF

Name Plate

Rating ( $\mathrm{KW}$ )

PESF

PESF*KW

Annual Energy Rating (MWh)

3700.00

$0.10 \quad 50.00$

Project

status

$\mathrm{XX}$

Dam
Status

Latitude

4006.00

Type

$$
\begin{aligned}
& \text { Type } \\
& \text { ROR }
\end{aligned}
$$

Factor

Exist Prob

Factor

Exist Prob

Wild/Scenic Protection

Wild/Scenic Tributary or Upstream/Downstream Wild/Scenic Location Cultural value Fish Presence Value Geologic Value Historic Value Other Value Recreation value Scenic Value
PAGE NO: 4

State

Name

OH

PESF Annual Energy Rating (MWh)

370

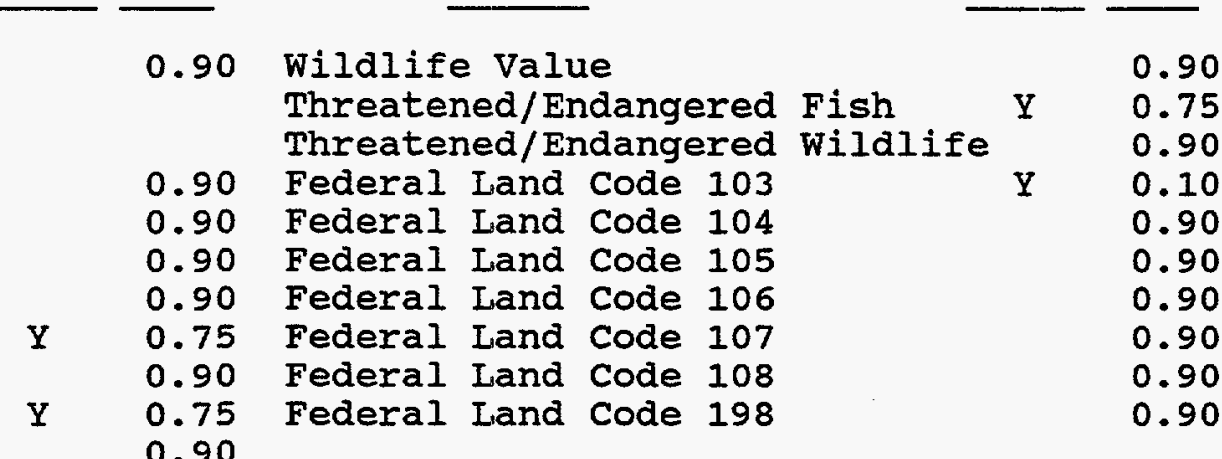


R E S O U R C E

DATE: $12 / 01 / 97$
D A T A B A S E

I I S T I N G

PAGE NO: 5

\section{FERC}

Number

Plant Name

03218 PIKE ISIAND

county Name

BEIMONT

Class Owner Name

M ORRVILLE, CITY OF

Name Plate

Rating ( $\mathrm{KW}$ )

PESF

Annual Energy

49500.00

$0.75 \quad 37125.00$

236400.00

River Basin

OHIO MAIN STREAM

$\begin{array}{cccccc}\text { Unit } & \text { Plant } & \text { Project } & \text { Dam } & \text { Latitude } & 4009.00 \\ \text { Type } & \text { Type } & \text { Status } & \text { Status } & \text { Longitude } 8042.00 \\ & \text { ROR } & \text { MO } & \text { Wo } & \end{array}$

Factor

Exist Prob

Factor

Exist Prob

Wild/scenic Protection wild/Scenic Tributary or Upstream/Downstream wild/Scenic Location cultural value Fish Presence Value Geologic Value Historic Value other Value Recreation Value Scenic Value

$\begin{array}{ll}0.90 & \text { Wildlife Value } \\ \text { Threatened/Endangered Fish } \\ \text { Threatened/Endangered wildlife } \\ 0.90 \text { Federal Land Code } 103 \\ 0.90 \text { Federal Land Code } 104 \\ 0.90 \text { Federal Land Code } 105 \\ 0.90 \text { Federal Land Code } 106 \\ 0.90 \text { Federal Land Code } 107 \\ 0.90 \text { Federal Land Code } 108 \\ 0.90 \text { Federal Land Code } 198 \\ 0.90\end{array}$

\subsection{Wildlife Value} Threatened/Endangered Fish Federal Land code 103

0.90 Federal Land Code 104 0.90 Federal Land code 105 0.90 Federal Land code 106 0.90 Federal Land code 107 0.90 Federal Land Code 198 0.90 state

Name

$\mathrm{OH}$
PESF Annual Energy Rating (MWh)

177300 
DATE: $12 / 01 / 97$

FERC

Number

03266

PIEDMONT DAM

County Name

HARRISON

Class

M

HARRISON COUNTY

Name Plate

Rating (KW)

PESF

PESF*KW

Annual Energy

300.00

$0.75 \quad 225.00$

2300.00

Stream

State

Name

STILLWATER CR,TUSCARAWAS $R$

$\mathrm{OH}$
River Basin

OHIO MAIN STREAM
PAGE NO: 6

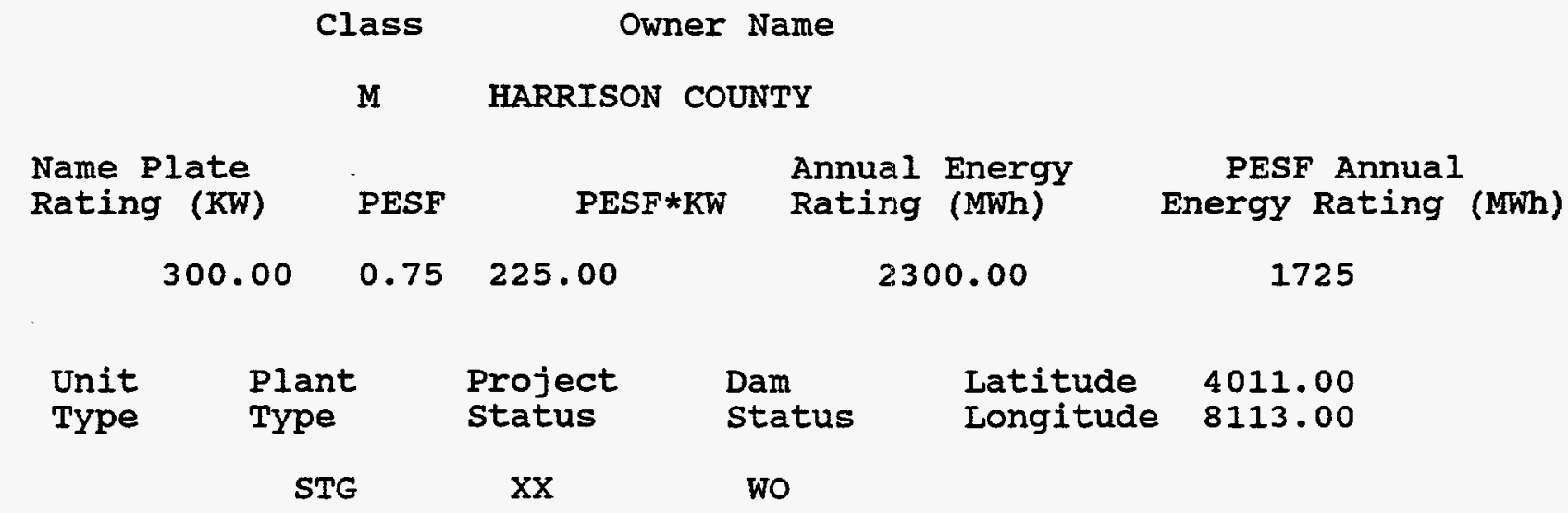

Factor

Exist Prob

Factor

Exist Prob

Wild/Scenic Protection

Wild/Scenic Tributary or

0.90 Wildlife Value

Threatened/Endangered Fish

0.90

Upstream/Downstream

Threatened/Endangered Wildlife

0.90

wild/Scenic Location

Cultural value

Fish Presence Value

$\begin{array}{ll}0.90 & \text { Federal Land Code } 103 \\ 0.90 & \text { Federal Land Code } 104\end{array}$

0.90

0.90

$\begin{array}{lll}0.90 & \text { Federal Land Code } 105 \\ 0.90 & \text { Federal Land Code } 106\end{array}$

0.90

0.75

0.90 Federal Land code 107

0.90

Historic Value

0.90

Federal Land Code 108

0.90

0.90

Federal Irand Code 198

0.90

0.90

Scenic Value 
DATE: $12 / 01 / 97$

FERC

Number

03279

KENT DAM

County Name

PORTAGE

Plant Name

Class

M KENT, CITY OF

Name Plate

Rating (KW)

PESF

PESF*KW

Annual Energy

420.00

$0.10 \quad 42.00$

Plant

Type

ROR

Factor

Exist Prob

Factor

Latitude

Dam
status

Longitude

4112.00

status

$\mathrm{XX}$

U
State

Name

$\mathrm{OH}$
PESF Annual Energy Rating (MWh)

192.60

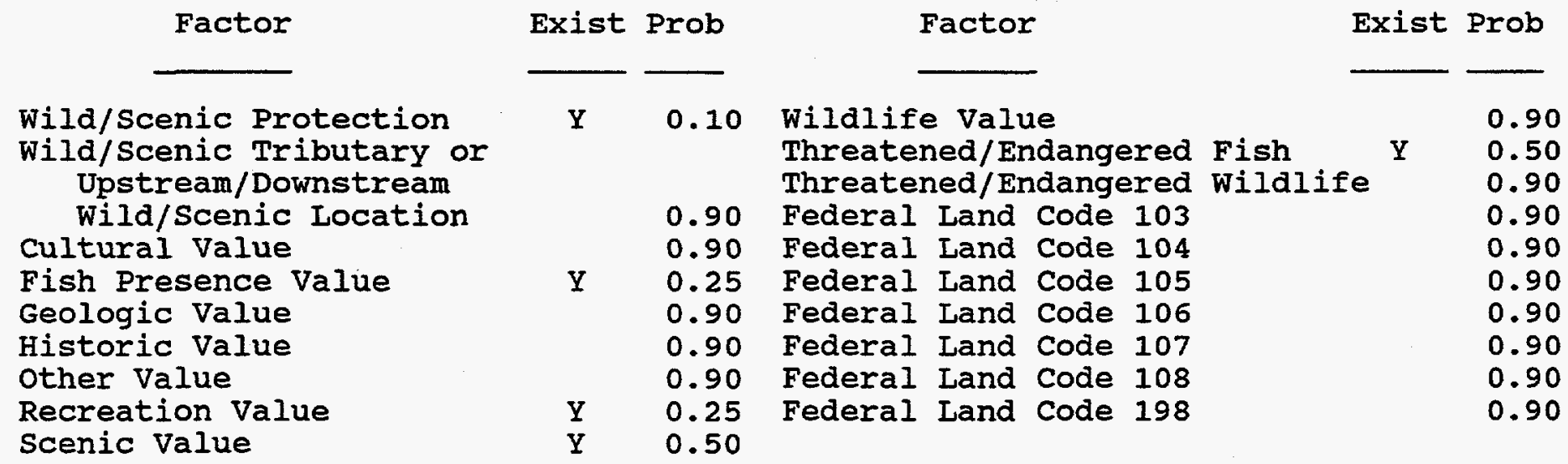


DATE: $12 / 01 / 97$

FERC

Number

03359

BERLIN LAKE

County Name

PORTAGE

Class

Owner Name

R CONTINENTAL HYDRO CORP

Name Plate

Rating (KW)

PESF

$\mathrm{PESF} * \mathrm{KW}$

Annual Energy

Rating (MWh)

5850.00

$0.75 \quad 1170.00$

1560.00

\begin{abstract}
Plant
Type

ROR
\end{abstract}

Unit

Factor

Exist Prob

0.90

Wild/Scenic Protection

Wild/Scenic Tributary or Upstream/Downstream wild/Scenic Location Cultural value

Fish Presence Value

Geologic Value

Historic Value

other Value

Recreation value

Scenic Value
River Basin

BEAVER RIVER BASIN
PAGE NO: 8

State

Name

$\mathrm{OH}$
PESF Annual Energy Rating (MWh)

4387.50 
R E S O U R C E

DATE: $12 / 01 / 97$
D A T A B A $\mathbf{E}$

FERC

Number

Plant Name

06074

DELAWARE DAM

County Name

DELAWARE

Class

Owner Name

R ENERGENICS SYSTEMS INC

Name Plate

Rating (KW)

PESF

Annual Energy

1200.00

0.50600 .00

PESF*KW

Rating (MWh)

PESF Annual

Energy Rating (MWh)

Unit Plant

Type Type

ROR

Project

status

XX

Exist Prob

Factor

wild/Scenic Protection

wild/Scenic Tributary or Upstream/Downstream wild/Scenic Location Cultural value Fish Presence Value

Geologic Value Historic Value other value Recreation Value scenic Value

PAGE NO: 9

State

Name

$\mathrm{OH}$
River Basin

OHIO MAIN STREAM 
DATE: $12 / 01 / 97$

PAGE NO: 10

FERC

Number

06075
ALUM CREEK

county Name

DELAWARE
Plant Name

$\mathbf{R}$

ENERGENICS SYSTEMS INC

Name Plate

Rating (KW)

PESF

Annual Energy

$\mathrm{PESF} * \mathrm{KW}$

Rating (MWh)

PESF Annual

900.00

0.1090 .00

1500.00

150

$\begin{array}{cc}\text { Unit } & \begin{array}{l}\text { Plant } \\ \text { Type }\end{array} \\ & \text { Rype } \\ \text { ROR }\end{array}$

state

Name

OH
Factor

wild/Scenic Protection

Wild/Scenic Tributary or Upstream/Downstream wild/Scenic Location

Cultural value

Fish Presence Value

Geologic Value

Historic Value

other value

Recreation Value

Scenic Value
Project status

XX

\section{Dam \\ status \\ Latitude \\ Longitude}

U
Exist Prob

Factor

4011.00

8257.00
Energy Rating (MWh)

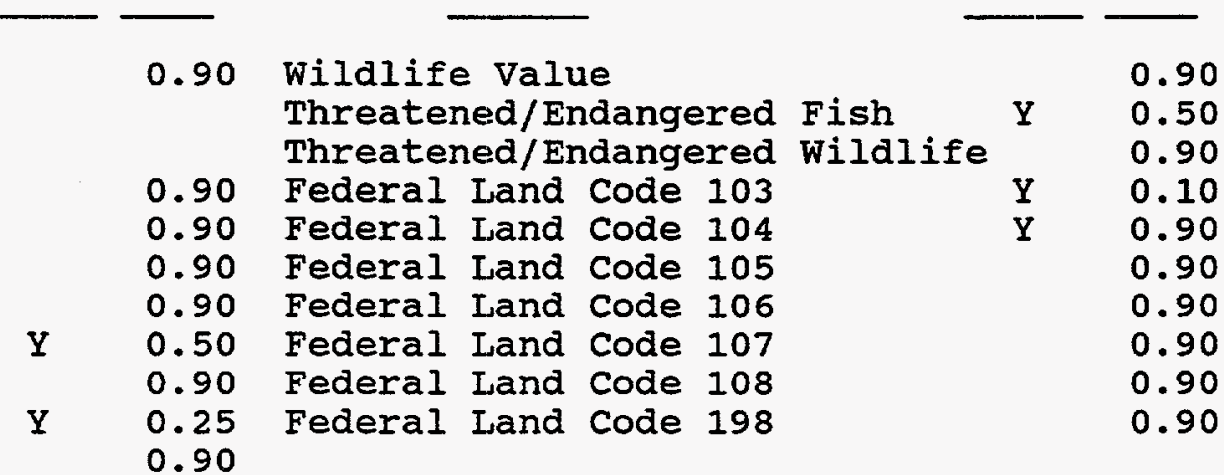


R E S O U R C E

DATE: $12 / 01 / 97$
D A T A B A S E

L I S T I N G

PAGE NO: 11

\section{FERC}

Number

Plant Name

06079

DEEP CREEK

County Name

PICKAWAY

\section{Class}

Owner Name

$R$ ENERGENICS SYSTEMS INC

Name Plate

Rating ( $\mathrm{KW}$ )

PESF

Annual Energy

2200.00

$0.10 \quad 220.00$

PESF*KW

Rating (MWh)
PESF Annual
Energy Rating (MWh)
480

4800.00

Unit

Plant

Project status

Dam

Status

Latitude

3937.00

Type

$$
\text { ROR }
$$

$\mathrm{XX}$

U

\section{Factor}

wild/scenic Protection

Wild/Scenic Tributary or Upstream/Downstream wild/Scenic Location Cultural value

Fish Presence Value

Geologic Value

Historic Value

other value

Recreation Value

Scenic Value
Exist Prob

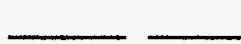

Wildife value Threatened/Endangered $F$ ish Threatened/Endangered Wildlife

Federal Land Code 103

0.90 Federal Land Code 104

0.90 Federal Land Code 105

0.90 Federal Land Code 106

Y $\quad 0.50$ Federal Land Code 107

0.90 Federal Land Code 108

Y 0.25 Federal Land Code 198

$$
0.90
$$

0.90

Factor

0.90
State

Name

$\mathrm{OH}$
Exist Prob

\begin{tabular}{lll} 
& & \\
\cline { 3 - 3 } Fish & & \\
Wildife & & 0.90 \\
& & 0.50 \\
& & 0.90 \\
& $Y$ & 0.10 \\
& & 0.90 \\
& & 0.90 \\
& & 0.90 \\
& & 0.90 \\
& & 0.90 \\
& & 0.90
\end{tabular}


DATE: $12 / 01 / 97$

PAGE NO: 12

FERC

Number

Plant Name

Stream

state

06563

MUSKINGUM L\&D 10

MUSKINGUM R

Name

county Name

River Basin

MUSKINGUM

OHIO MAIN STREAM

Class Owner Name

R ENERGENICS SYSTEMS INC

Name Plate

Rating (KW)

PESF

Annual Energy

930.00

$0.75 \quad 697.50$

4300.00

OH

PESF Annual Energy Rating (MWh)

3225

$\begin{array}{cccccc}\text { Unit } & \text { Plant } & \text { Project } & \text { Dam } & \text { Latitude } & 3956.00 \\ \text { Type } & \text { Type } & \text { Status } & \text { Status } & \text { Longitude } & \mathbf{8 1 0 1 . 0 0} \\ & \text { ROR } & \mathrm{XX} & \text { Wo } & \end{array}$

\author{
Factor \\ Wild/Scenic Protection \\ wild/Scenic Tributary or \\ Upstream/Downstream \\ wild/Scenic Location \\ Cultural Value \\ Fish Presence Value \\ Geologic Value \\ Historic Value \\ other Value \\ Recreation Value \\ Scenic Value
}

Factor

Exist Prob

\subsection{Wildife Value}

Threatened/Endangered Fish

Threatened/Endangered wildife

0.90 Federal Iand code 103

0.90 Federal Land code 104

0.90 Federal Land Code 105

0.90 Federal Iand Code 106

0.90 Federal Land Code 107

0.90 Federal Iand code 108

0.90 Federal Land code 198

0.90

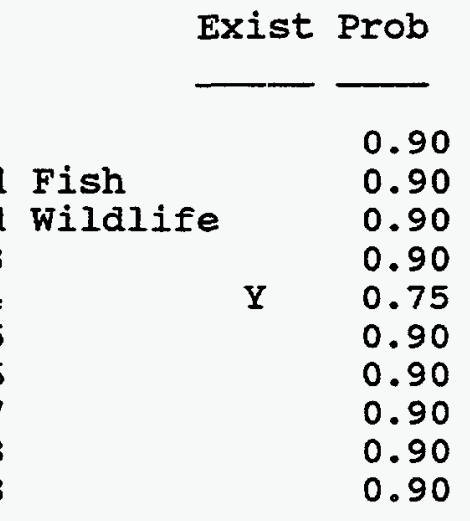


DATE: $12 / 01 / 97$

FERC

Number

06637
Plant Name

DEFIANCE (AUGLAIZE R)

County Name

DEFIANCE
PAGE NO: 13

state

Name

AUGLAIZE R

$\mathrm{OH}$
River Basin

MAUMEE RIVER BASIN

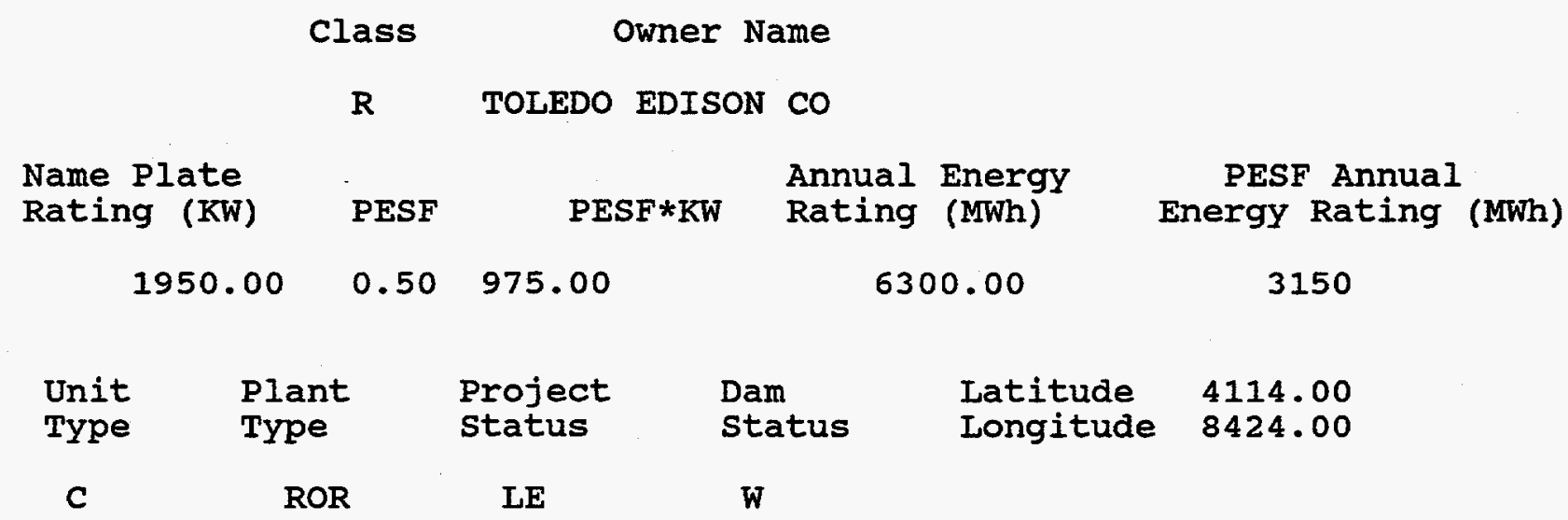

Factor

Wild/Scenic Protection Wild/Scenic Tributary or Upstream/Downstream wild/Scenic Location Cultural value Fish Presence Value Geologic Value Historic Value other value Recreation Value Scenic Value
Exist Prob

0.90 Wildlife Value Threatened/Endangered Fish Threatened/Endangered Wildiife

Exist Prob

0.90

Federal Land Code 103

0.90 Federal Land Code 104

Y $\quad 0.75$ Federal Land Code 105

0.90 Federal Land Code 106

$Y \quad 0.75$ Federal Land Code 107

$\mathrm{Y} \quad 0.75$ Federal Land Code 108

$Y \quad 0.75$ Federal Land Code 198

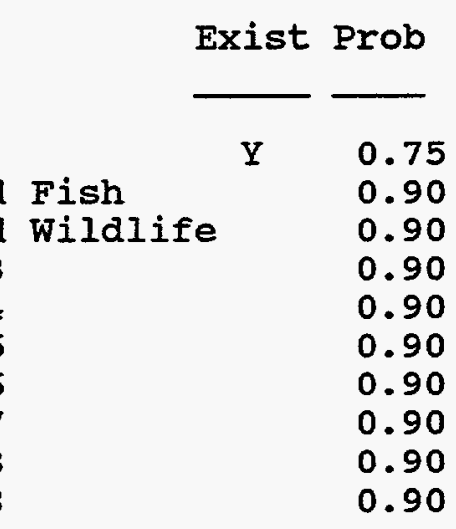


DATE: $12 / 01 / 97$

PAGE NO: 14

FERC

Number

06998 MUSKINGUM L\&D 3

county Name

WASHINGTON

\section{Class}

R UPPER MISSISSIPPI WTR CO

Name Plate Rating ( $K W$ )

10000.00

PESF

0.909000 .00
Project status

XX
Annual Energy Rating (MWh)

50000.00 state

Name

O.H

$\begin{array}{cc}\text { Unit } & \begin{array}{l}\text { Plant } \\ \text { Type }\end{array} \\ & \text { RORe }\end{array}$

Factor

wild/Scenic Protection wild/Scenic Tributary or Upstream/Downstream wild/Scenic Location Cultural value Fish Presence Value Geologic Value Historic Value other value Recreation Value Scenic Value
Dam
Status

WO
Exist Prob

Factor

Iatitude Longitude
PESF Anniual Energy Rating (MWh) 45000

3932.00

8131.00 
R E S O U R E D A T A B A S E L I S T I N G

DATE: $12 / 01 / 97$

PAGE NO: 15

FERC

Number

Plant Name

Stream

State

Name

07220 ELLIS L\&D 11

MUSKINGUM $R$

$\mathrm{OH}$

county Name

River Basin

MUSKINGUM

OHIO MAIN STREAM

$$
\begin{array}{cc}
\text { Class } & \text { Owner Name } \\
\text { R } & \text { MUSKINGUM R HYDRO ASSOC }
\end{array}
$$

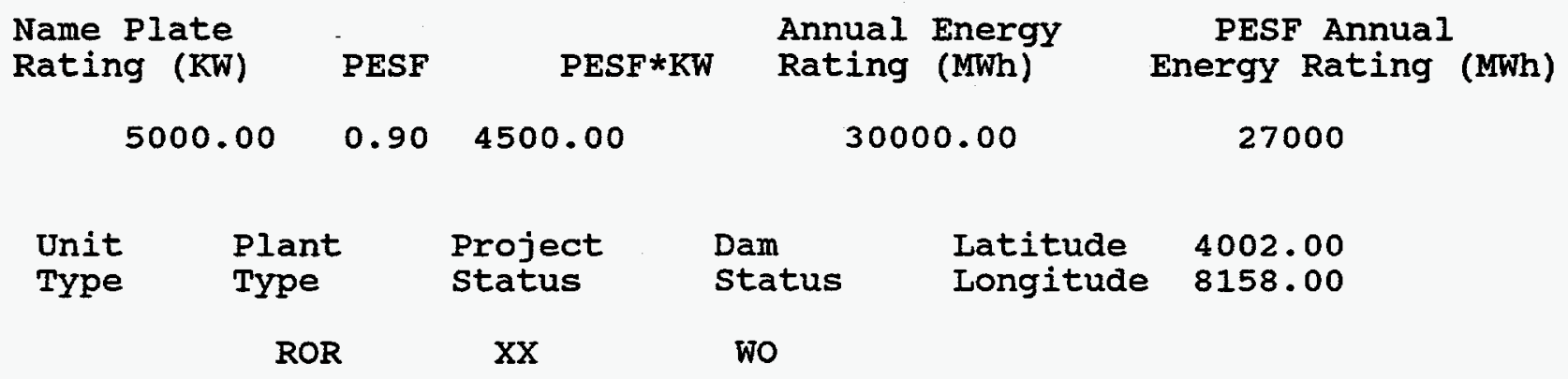

Factor

Wild/Scenic Protection

wild/Scenic Tributary or Upstream/Downstream wild/Scenic Location Cultural Value

Fish Presence Value

Geologic Value

Historic Value

other Value

Recreation Value

Scenic Value
Exist Prob

0.90 Wildlife Value

Threatened/Endangered Fish

Threatened/Endangered Wildlife

0.90 Federal Land code 103

0.90 Federal Land Code 104

0.90 Federal Land Code 105

0.90 Federal Land Code 106

0.90 Federal Land Code 107

0.90 Federal Land Code 108

0.90 Federal Land Code 198

0.90
Exist Prob

0.90

0.90

0.90

0.90

0.90

0.90

0.90

0.90

0.90

0.90 
R E S O U R C E

DATE: $12 / 01 / 97$

FERC

Number

Plant Name

07221 PHILO I\&D 9

county Name

MUSKINGUM

\section{Class}

R

MUSKINGUM R HYDRO ASSOC

Name Plate

Rating ( $K W$ )

PESF

PESF*KW

Annual Energy

5800.00

$0.90 \quad 5220.00$

MUSKINGUM $\mathbf{R}$

stream

River Basin

OHIO MAIN STREAM
I I S T N G

PAGE NO: 16

State

Name

$\mathrm{OH}$
PESF Annual Energy Rating (MWh)

$\begin{array}{cccccc}\text { Unit } & \text { Plant } & \text { Project } & \text { Dam } & \text { Latitude } & 3952.00 \\ \text { Type } & \text { Type } & \text { Status } & \text { Status } & \text { Longitude } & 8155.00 \\ & \text { ROR } & \mathrm{XX} & \text { WO } & \end{array}$

Factor

Exist Prob

Factor

Exist Prob

Wild/Scenic Protection

wild/Scenic Tributary or

0.90 wildlife value

0.90

Threatened/Endangered Fish

0.90

Upstream/Downstream

wild/Scenic Location

Threatened/Endangered Wildlife

0.90

0.90 Federal Land Code 103

0.90

0.90 Federal Land Code 104

0.90

0.90 Federal Land Code 105

0.90

0.90 Federal Land Code 106

0.90

0.90 Federal Land Code 107

0.90

Historic Value

other value

Recreation Value

0.90 Federal Land Code 108

0.90

0.90

Federal Land Code 198

0.90

Scenic value

0.90 
R E S O U R C E

DATE: 12/01/97
D A T A B A S E

FERC

Number

07222
Plant Name

ROKEBY I\&D 8

county Name

MORGAN
PAGE NO: 17

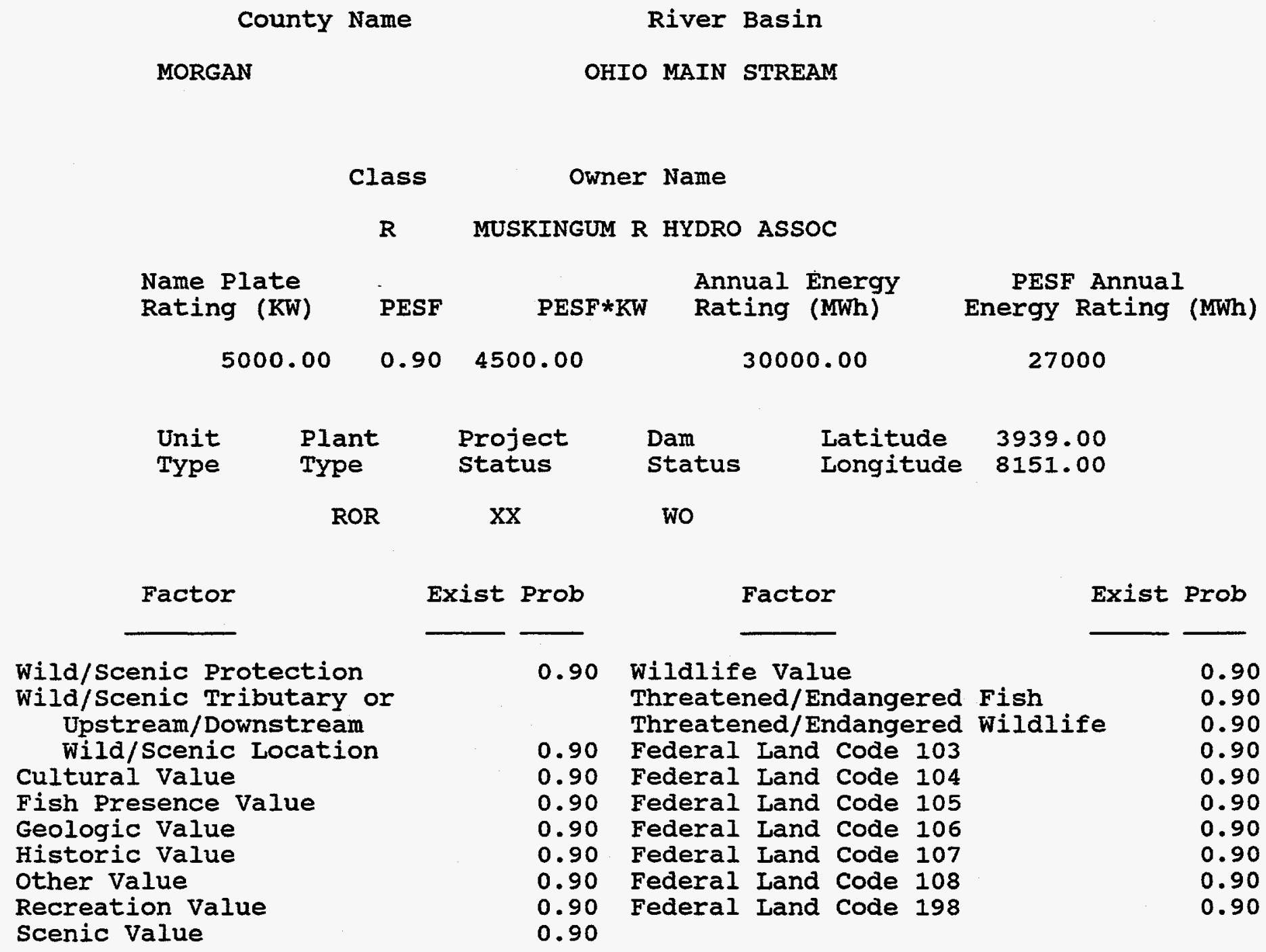

state

Name

OH

\author{
MUSKINGUM R
}

stream

L I S T I N G

PAGE NO: 17 
DATE: $12 / 01 / 97$

PAGE NO: 18

FERC

Number

07234
Plant Name

BEVERLY I\&D 4

county Name

WASHINGTON

\section{stream}

MUSKINGUM $R$

River Basin

OHIO MAIN STREAM state

Name

$\mathrm{OH}$

$$
\begin{array}{cc}
\text { Class } & \text { Owner Name } \\
\text { R } & \text { MUSKINGUM R HYDRO ASSOC }
\end{array}
$$

Name Plate

Rating ( $\mathrm{KW}$ )

5000.00
Annual Energy PESF*KW
Rating (MWh)

30000.00
PESF Annua1 Energy Rating (MWh)

$\begin{array}{ccccc}\text { Unit } & \text { Plant } & \text { Project } & \text { Dam } & \text { Latitude } \\ \text { Type } & \text { Type } & \text { Status } & \text { Status } & \text { Longitude } \\ & \text { ROR } & \text { XX } & \text { wo }\end{array}$

Factor

Exist Prob

Factor

Exist Prob

wild/scenic Protection

wild/scenic Tributary or Upstream/Downstream wild/Scenic Location

\subsection{Wildife Value}

Threatened/Endangered Fish Threatened/Endangered Wildlife

27000

Cultural value

Fish Presence Value

$\begin{array}{ll}0.90 & \text { Federal Land Code } 103 \\ 0.90 & \text { Federal Land Code } 104\end{array}$

3933.00

8139.00

Geologic Value

0.90 Federal Land Code 105

0.90 Federal Land Code 106

0.90 Federal Land Code 107

0.90 Federal Land Code 108

other Value

Recreation value

0.90

Federal Land Code 198

0.90 
DATE: $12 / 01 / 97$

FERC

Number

Plant Name

07237 ELYRIA

County Name

LORAIN

Class

R MULLER, EUGENE F

Name Plate

Rating (KW)

PESF

Annual Energy

650.00

$0.50 \quad 325.00$

$\mathrm{PESF} * \mathrm{KW}$

Rating (MWh)

2456.00

Unit

Plant

Type

Type

ROR

Factor

Exist Prob

Wild/Scenic Protection

wild/Scenic Tributary or

Upstream/ Downstream

wild/Scenic Location

Cultural Value

Fish Presence Value

Geologic Value

Historic Value

other Value

Recreation value

Scenic Value
PAGE NO: 19

State

Name

$\mathrm{OH}$
PESF Annual Energy Rating (MWh)

1228 $\begin{array}{lll}\text { Dam } & \text { Latitude } & \mathbf{4 1 2 2 . 0 0} \\ \text { Status } & \text { Longitude } & \mathbf{8 2 0 6 . 0 0}\end{array}$

wo
0.90

wildife Value Threatened/Endangered Fish Threatened/Endangered Wildlife

0.90 Federal Land Code 103

0.90 Federal Land Code 104

0.90 Federal Land Code 105

0.90 Federal Land Code 106

$\mathrm{Y} \quad 0.75$

Federal Land Code 107

Federal Land Code 108

0.90

Federal Land Code 198
Exist Prob

0.90

0.90

0.90

0.90

0.90

0.90

0.90

0.90

0.90

0.90 
DATE: $12 / 01 / 97$

PAGE NO: 20

FERC

Number

07261
Plant Name

IUKE CHUTE I\&D 5

county Name

MORGAN

\section{Stream}

MUSKINGUM R

River Basin

OHIO MAIN STREAM
St:ate

Name

CII

\section{Class Owner Name \\ R MUSKINGUM R HYDRO ASSOC}

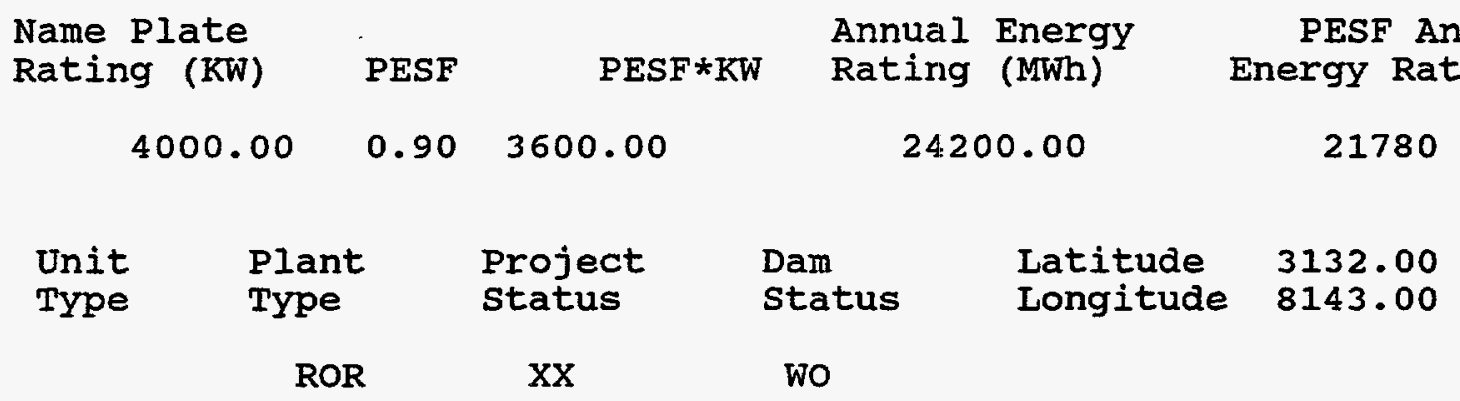

Factor

Wild/scenic Protection

Wild/Scenic Tributary or Upstream/Downstream wild/Scenic Location Cultural Value Fish Presence Value Geologic Value Historic Value other Value Recreation value Scenic Value
Exist Prob

Factor

0.90 Wildlife Value

Threatened/Endangered Fish Threatened/Endangered Wildlife

0.90 Federal Land code 103

0.90 Federal Land Code 104

0.90 Federal Land code 105

0.90 Federal Land code 106

0.90 Federal Land code 107

0.90 Federal Land Code 108

0.90 Federal Land code 198

0.90
Exist Prob

$\begin{array}{ll} & \text { Exist Prob } \\ & \\ & \\ \text { Fish } & 0.90 \\ \text { Wildife } & 0.90 \\ & 0.90 \\ & 0.90 \\ & 0.90 \\ & 0.90 \\ & 0.90 \\ & 0.90 \\ & 0.90 \\ & 0.90\end{array}$

0.90

0.90

0.90

0.90

0.90 
R E S O U R C E

DATE: $12 / 01 / 97$
D A T A B A S E

L I S T I N G

FERC

Number

Plant Name

07262 MCCONNELSVILLE L\&D 7

County Name

MORGAN

PAGE NO: 21

stream

State

Name

MUSKINGUM $\mathrm{R}$

OH

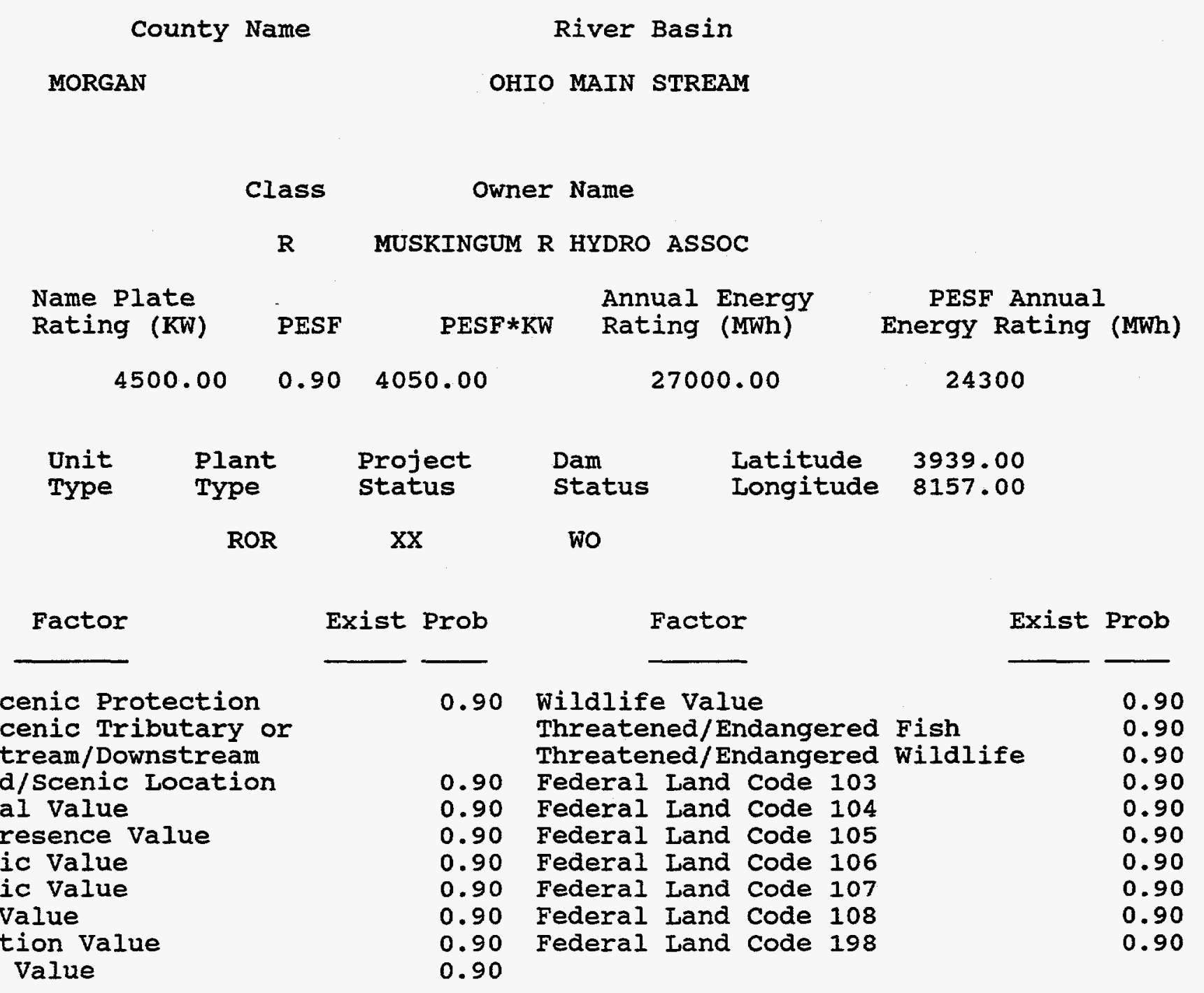

Wild/Scenic Protection

wild/Scenic Tributary or Upstream/Downstream wild/Scenic Location Cultural Value Fish Presence Value Geologic Value Historic Value other Value Recreation value Scenic Value
0.90
0.90

0.90

0.90

0.90

0.90

0.90

0.90 
FERC

Number

07751
Plant Name

DEVOIAA L\&D 2

county Name

WASHINGTON

Class

R MUSKINGUM R HYDRO ASSOC

Name Plate

Rating ( $\mathrm{KW}$ )

PESF

PESF*KW

Annual Energy

Rating (MWh)

7300.00

0.90

6570.00

23200.00
River Basin

OHIO MAIN STREAM state

Name

$\mathrm{OH}$

$\begin{array}{cccccc}\text { Unit } & \text { Plant } & \text { Project } & \text { Dam } & \text { Latitude } & 3928.00 \\ \text { Type } & \text { Type } & \text { Status } & \text { Status } & \text { Longitude } & 8130.00 \\ & \text { ROR } & \mathrm{XX} & \text { wo } & \end{array}$

Factor

Exist Prob

Factor

Exist Prob

Wild/Scenic Protection

wild/Scenic Tributary or

0.90 Wildlife Value

Threatened/Endangered Fish

PESF Annual Energy Rating (MWh)

Upstream/Downstream

wild/Scenic Location

Threatened/Endangered wildlife

0.90

$\begin{array}{ll}0.90 & \text { Federal Land Code } 103 \\ 0.90 & \text { Federal Land Code } 104\end{array}$

0.90

0.90

Cultural value

0.90 Federal Land Code 105

0.90

0.90 Federal Land code 106

0.90

Fish Presence Value

0.90 Federal Land Code 107

0.90

Historic Value

0.90

Federal Land Code 108

0.90

Other Value

Recreation Value

0.90

Federal Land Code 198

0.90

0.90

Scenic Value

0.90

0.90 
$\begin{array}{lllllll}R & E & S & O & \mathrm{U} & \mathrm{C} & \mathrm{E}\end{array}$

DATE: $12 / 01 / 97$
D A T A B A $S E$

PAGE NO: 23
FERC

Number

07871
Plant Name

PAINT CREEK

county Name

ROSS

Class

Owner Name

R ROSS ASSOC

Name Plate

Rating (KW)

PESF

PESF*KW

Annual Energy

$$
8000.00
$$

$0.50 \quad 4000.00$

Rating (MWh)

$$
35000.00
$$

Dam

status

Latitude

Longitude

status

XX

wo
State

Name

$\mathrm{OH}$

$\begin{array}{cccccc}\text { Unit } & \text { Plant } & \text { Project } & \text { Dam } & \text { Latitude } & 3915.00 \\ \text { Type } & \text { Type } & \text { Status } & \text { Status } & \text { Longitude } & 8321.00 \\ & \text { ROR } & \mathrm{XX} & \text { wo } & & \end{array}$

Factor

Wild/Scenic Protection Wild/Scenic Tributary or Upstream/Downstream wild/scenic Location Cultural Value Fish Presence Value Geologic Value Historic Value other Value Recreation value Scenic Value
Exist Prob

Factor

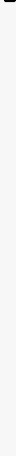

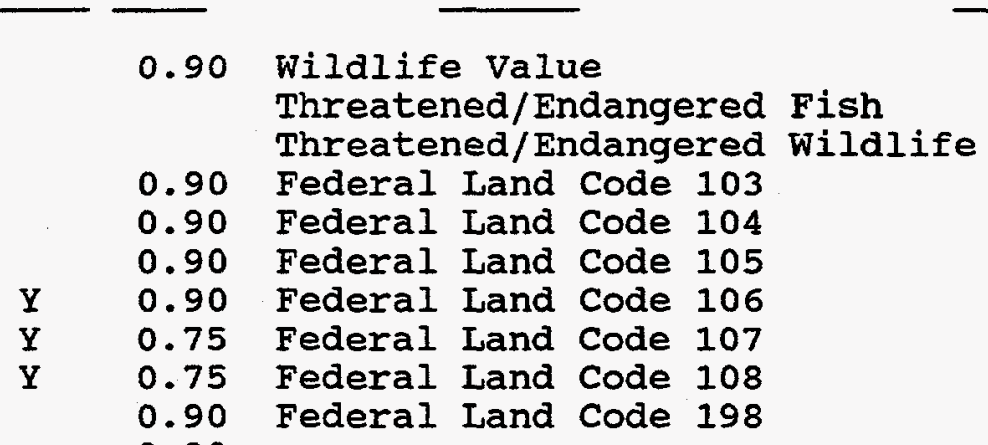

0.90
Exist Prob

PESF Annual Energy Rating (MWh)

17500

$\begin{array}{ll} & 0.90 \\ Y \quad & 0.75 \\ 0.90 \\ 0.90 \\ \\ Y \quad 0.75 \\ 0.90 \\ 0.90 \\ 0.90 \\ 0.90 \\ 0.90\end{array}$


DATE: $12 / 01 / 97$

PAGE NO: 24

FERC

Number

07933
CAESAR CREEK

county Name

WARREN
Plant Name
State

Name

CAESAR CR, LITTLE MIAMI $R$

$\mathrm{OH}$
River Basin

MINOR RIVER BASINS

\section{Class Owner Name}

R WARREN HYDRO PARTNERS

\begin{tabular}{|c|c|c|c|c|c|c|c|}
\hline \multicolumn{2}{|c|}{$\begin{array}{l}\text { Name Plate } \\
\text { Rating (KW) }\end{array}$} & PESF & PESF*KW & $\begin{array}{l}\text { Ann } \\
\text { Rat }\end{array}$ & $\begin{array}{l}\text { Energy } \\
\text { (MWh) }\end{array}$ & $\begin{array}{l}\text { PESF Annual } \\
\text { Energy Rating }\end{array}$ & (MWh) \\
\hline \multicolumn{2}{|c|}{3000.00} & 0.50 & 1500.00 & \multicolumn{2}{|c|}{12000.00} & 6000 & \\
\hline \multirow[t]{2}{*}{$\begin{array}{l}\text { Unit } \\
\text { Type }\end{array}$} & \multicolumn{2}{|c|}{$\begin{array}{l}\text { Plant } \\
\text { Type }\end{array}$} & $\begin{array}{l}\text { Project } \\
\text { Status }\end{array}$ & $\begin{array}{l}\text { Dam } \\
\text { status }\end{array}$ & $\begin{array}{l}\text { Latitude } \\
\text { Longitude }\end{array}$ & $\begin{array}{l}3928.00 \\
8403.00\end{array}$ & \\
\hline & \multicolumn{2}{|c|}{ ROR } & $X X$ & wo & & & \\
\hline
\end{tabular}

Factor

wild/Scenic Protection

wild/Scenic Tributary or Upstream/Downstream wild/Scenic Location Cultural value

Fish Presence Value

Geologic Value

Historic Value

other Value

Recreation value

Scenic Value
Exist Prob

Factor

$\mathrm{Y} \quad 0.50$

Wildlife Value

Threatened/Endangered Fish Threatened/Endangered wildiife

0.90 Federal Land code 103

0.90 Federal I and code 104

0.90 Federal Land Code 105

0.90 Federal Land code 106

0.90 Federal Land code 107

0.90 Federal Land Code 108

0.90 Federal Land code 198

0.90
Exist Prob

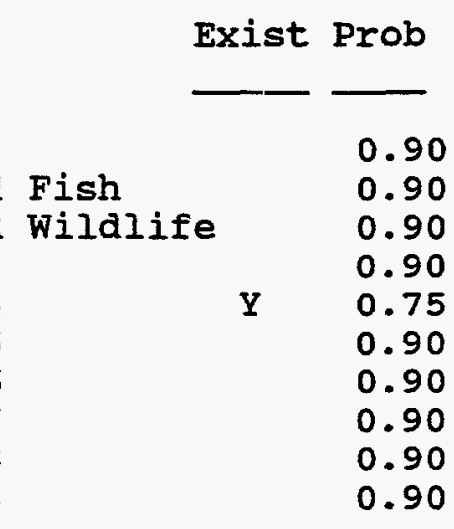

0.90

0.90

0.75

0.90

0.90 
R E S O U R C E

DATE: $12 / 01 / 97$
D A T A B A S E

L I S T I N G

PAGE NO: 25

FERC

Number

Plant Name

stream

State

08268

DILION

IICKING $\mathrm{R}$

$\mathrm{OH}$

County Name

River Basin

MUSKINGUM

OHIO MAIN STREAM

\author{
Class Owner Name \\ $R$ DILLON LAKE HYDRO ASSOC
}

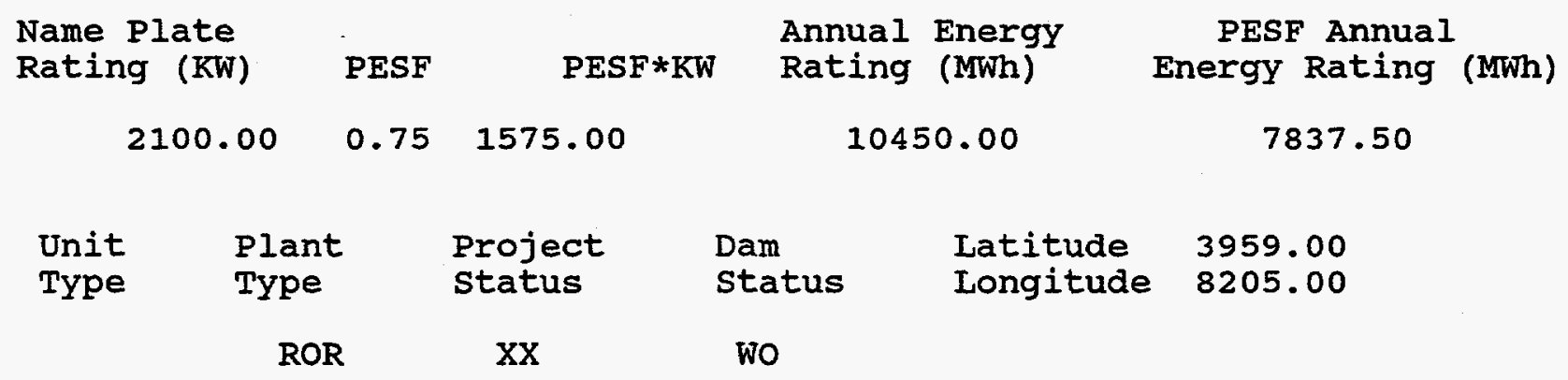

Factor

Exist Prob

Factor

Exist Prob

Wild/Scenic Protection

wild/Scenic Tributary or

0.90 Wildlife Value
Threatened/Endangered Fish

0.90

Upstream/Downstream Threatened/Endangered Wildlife

0.90

wild/Scenic Location

0.90 Federal Land Code 103

0.90

Cultural Value

0.90 Federal Land Code 104

0.90

Fish Presence Value

0.90 Federal Land Code 105

0.75

0.90 Federal Land Code 106

0.90

Geologic Value

0.90 Federal Land Code 107

0.90

Historic Value

0.90 Federal Land Code 108

0.90

other Value

0.90 Federal Land Code 198

0.90

Recreation Value

0.90

0.90 
DATE: $12 / 01 / 97$

PAGE NO: 26

FERC

Number

08269
Plant Name

WILLS CREEK

County Name

COSHOCTON

Class

Name Plate

Rating ( $K W$ )

2400.00
$\mathbf{R}$

WILIS CREEK HYDRO ASSOC

PESF

PESF*KW

Annual Energy

Rating (MWh)

$0.75 \quad 1800.00$

10300.00

River Basin

OHIO MAIN STREAM

Project
Status
XX

Exist Prob

Factor

Wild/Scenic Protection

Wild/Scenic Tributary or Upstream/Downstream wild/Scenic Location Cultural Value

Fish Presence Value

Geologic Value

Historic Value

other value

Recreation Value

Scenic Value

$\begin{array}{lll}\text { Dam } & \text { Latitude } & 4006.00 \\ \text { Status } & \text { Longitude } & 8151.00 \\ & \end{array}$

Factor

Exist Prob
St:ate

Name

$\mathrm{OH}$
PESF Anriual

7725

(MWh) 
R E S O U R E

DATE: $12 / 01 / 97$
D A T A B A $S E$
L I S T I N G

FERC

Number

Plant Name

stream

state

Name

08270

PLEASANT HIIL

County Name

CLEAR FORK R

$\mathrm{OH}$

ASHLAND

River Basin

OHIO MAIN STREAM

Class Owner Name

R PLEASANT HILL HYDRO ASSOC

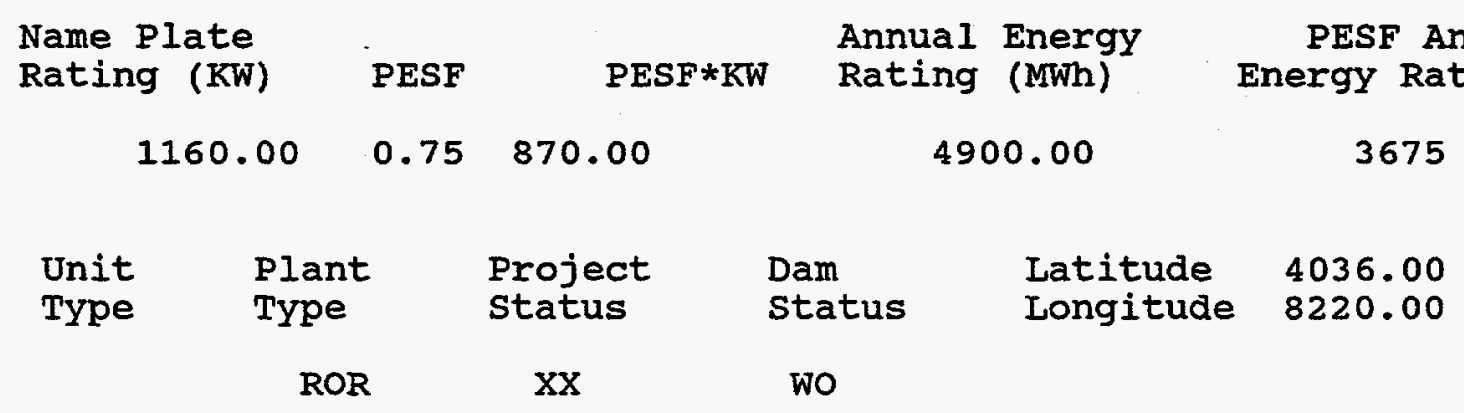

Factor

Wild/Scenic Protection

wild/Scenic Tributary or

Upstream/ Downstream

wild/Scenic Location

Cultural value

Fish Presence Value

Geologic Value

Historic Value

other Value

Recreation Value

Scenic value
Exist Prob

0.90

Wildlife Value

Threatened/Endangered Wildilife

0.90 Federal Land Code 103

0.90 Federal Land Code 104

0.90 Federal Land Code 105

0.90 Federal Land Code 106

0.90 Federal Land Code 107

0.90 Federal Land Code 108

0.90 Federal Land Code 198

0.90

Exist Prob

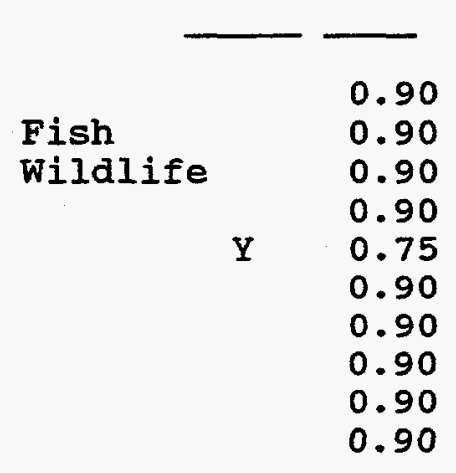


DATE: $12 / 01 / 97$

PAGE NO: 28

FERC

Number

Plant Name

stream

Stiate

08376 MUSKINGUM L\&D 6

MUSKINGUM R

Name

county Name

River Basin

MORGAN

OHIO MAIN STREAM

Class Owner Name

I STOCKPORT MILIING CO, INC

Name Plate

PESF

Annual Energy

Rating (KW)

$0.90 \quad 1350.00$

Rating (MWh)

PESF Annual

1500.00

12000.00

Energy Rating (MWh)

Unit

$$
\begin{aligned}
& \text { Plant } \\
& \text { Type } \\
& \text { ROR }
\end{aligned}
$$

10800

$\mathrm{OH}$

Factor

Wild/Scenic Protection

wild/Scenic Tributary or Upstream/Downstream wild/Scenic Location

cultural value

Fish Presence Value

Geologic Value

Historic Value

other value

Recreation value

Scenic Value
Project

status

Dam

status

Latitude

$\mathrm{xX}$

wo

Exist Prob

Factor

Exist Prob

3933.00

Longitude 8147.00
0.90 Wildlife Value

Threatened/Endangered Fish

0.90

0.90

0.90

0.90 Federal Land Code 103

0.90 Federal Land Code 104

0.90 Federal Land Code 105

0.90 Federal Land Code 106

0.90 Federal Land Code 107

0.90 Federal Land Code 108

0.90 Federal Land Code 198

0.90

0.90

0.90

0.90

0.90

0.90

0.90

0.90 

R E S O U R C E
D A T A B A S E
L I S T I N G

DATE: $12 / 01 / 97$

PAGE NO: 29

FERC

Number

Plant Name

Stream

state

09042

GALLIPOLIS L\&D

OHIO $\mathrm{R}$

Name

county Name

River Basin

GALIIA

OHIO MAIN STREAM

Class Owner Name

R GALIIA HYDRO PARTNERS

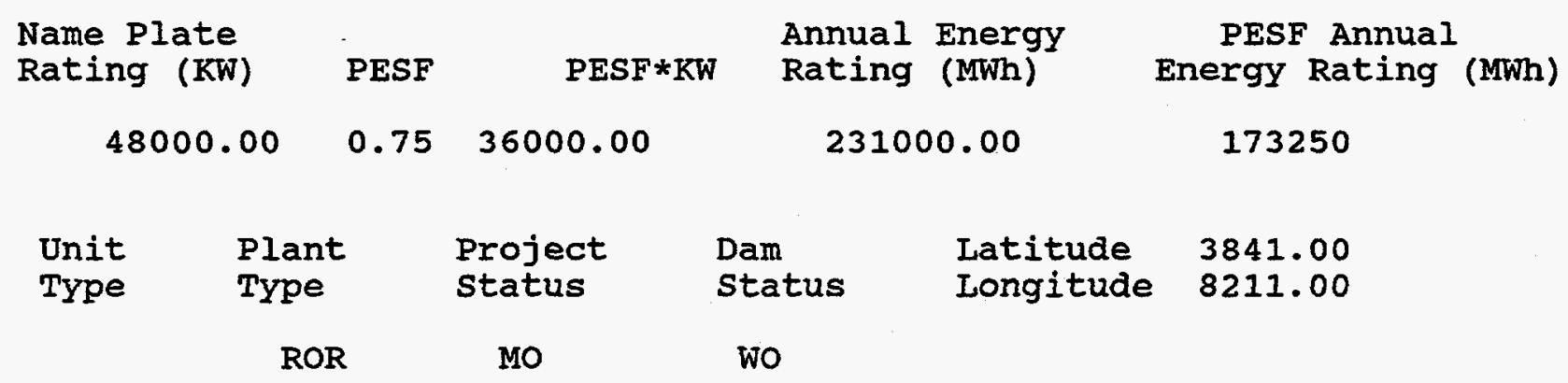

Factor

Exist Prob

Factor

Exist Prob

wild/scenic Protection

wild/Scenic Tributary or

Upstream/Downstream

wild/Scenic Location

0.90
0.90
0.90
0.90
0.90
0.90
0.90
0.90
0.90

Wildlife Value

Threatened/Endangered Fish

Threatened/Endangered Wildlife

OH

cultural value

Fish Presence Value

Federal Land code 103

Federal Land code 104

Federal Land code 105

Federal Irand code 106

Federal Land code 107

Federal Land Code 108

Federal Land code 198

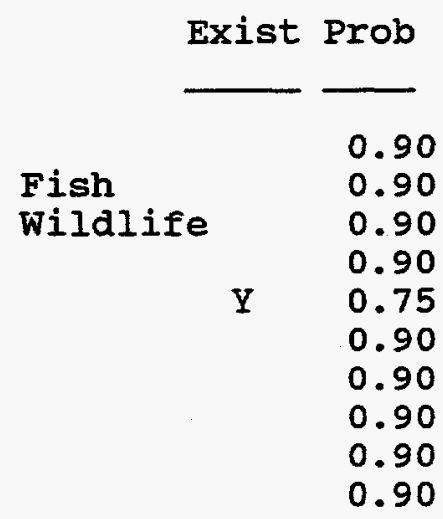

Scenic Value

0.90
0.90

0.90 
DATE: $12 / 01 / 97$

PAGE NO: 30

FERC

Number

Plant Name

Stream

St:ate

Name

09972 MILTON

MAHONING $R$

$\mathrm{OH}$

County Name

MAHONING

Class

M YOUNGSTOWN, CITY OF

Name Plate

Rating (KW)

PESF

Annual Energy

PESF*KW
PESF Annual Energy Rating (MWh)

$$
1000.00 \quad 0.90 \quad 900.00
$$

$$
4.000 .00
$$

3600

$\begin{array}{cccccc}\text { Unit } & \text { Plant } & \text { Project } & \text { Dam } & \text { Latitude } & 4107.00 \\ \text { Type } & \text { Type } & \text { Status } & \text { Status } & \text { Longitude } & 8058.00 \\ & \text { ROR } & \mathrm{XX} & \text { WO } & \end{array}$

Factor

Exist Prob

Factor

Exist Prob

Wild/Scenic Protection

wild/Scenic Tributary or

0.90 Wildife Value

Threatened/Endangered Fish

Upstream/Downstream Threatened/Endangered Wildlife

0.90

wild/Scenic Location

0.90

Federal Land Code 103

0.90 Federal Land Code 104

0.90 Federal Land Code 105

0.90 Federal Land Code 106

0.90 Federal Land Code 107

0.90 Federal Land Code 108

0.90 Federal Land Code 198

$\begin{array}{ll} & \\ & \\ \text { Fish } & 0.90 \\ \text { Wildife } & 0.90 \\ & 0.90 \\ & 0.90 \\ & 0.90 \\ & 0.90 \\ & 0.90 \\ & 0.90 \\ & 0.90 \\ & 0.90\end{array}$

0.90

Scenic Value 
DATE: $12 / 01 / 97$

FERC

Number

10252
Plant Name

BOOSTER STA

County Name

MAHONING
PAGE NO: 31

State

Name

OWSC PL(MAHONING R)

$\mathrm{OH}$
River Basin

BEAVER RIVER BASIN

\section{Class Owner Name}

R OHIO WTR SERVICE CO INC

Name Plate

Rating (KW)

200.00
PESF

$0.90 \quad 180.00$

Unit

Type

Plant
Type

DIV

Factor

Wild/Scenic Protection

Wild/Scenic Tributary or Upstream/Downstream wild/Scenic Location Cultural Value Fish Presence Value Geologic Value Historic Value other Value Recreation Value Scenic Value
Project status

$\mathrm{xx}$

Exist Prob

0.90

0.90

0.90

0.90

0.90

0.90

0.90

0.90

0.90
Annual Energy Rating (MWh)

1700.00

Latitude Longitude
PESF Annual Energy Rating (MWh) 1530
Dam Status

U
4110.00

8042.00 
DATE: $12 / 01 / 97$

FERC

Number

10619

CUYAHOGO FALLS

County Name

SUMMIT

Class

Owner Name

R CUYAHOGA FALLS HYDRO ASSOC

Name Plate

Rating (KW)

PESF

Annual Energy

5500.00

$0.50 \quad 2750.00$

24000.00

Latitude

Unit

$$
\begin{aligned}
& \text { Plant } \\
& \text { Type } \\
& \text { ROR }
\end{aligned}
$$

Factor

Exist Prob

Exist Prob

wild/Scenic Protection

wild/Scenic Tributary or Upstream/Downstream wild/Scenic Location

Cultural Value

Fish Presence Value

Geologic Value

Historic Value

Other Value

Recreation value

Scenic Value
Longitude

4108.00

Factor

$\begin{array}{cll}\begin{array}{c}\text { Project } \\ \text { status }\end{array} & \begin{array}{l}\text { Dam } \\ \text { status }\end{array} & \begin{array}{l}\text { Latitude } \\ \text { Longitude }\end{array} \\ \mathrm{xx} & \text { wo } & \end{array}$

$\mathrm{Y} \quad 0.50$

Wildife Value

Threatened/Endangered Fish Threatened/Endangered Wildlife

0.90 Federal Land Code 103

$Y \quad 0.75$ Federal Land Code 105

0.90 Federal Land Code 106

0.90 Federal Land Code 107

0.90 Federal Land Code 108

$\mathrm{Y} \quad 0.75$ Federal Land Code 198

$\Psi \quad 0.90$
0.90 Federal Irand Code 104
State

Name

$\mathrm{OH}$
PESF Anrual Energy Rating (MWh)

12000 
FERC

Number

10860
Plant Name

EAST FORK

county Name

CLERMONT stream

E FK LITTLE MIAMI R

River Basin

MINOR RIVER BASINS
State

Name

$\mathrm{OH}$

\section{Class Owner Name}

$R$ LEWIS, WM \& ASSOC INC

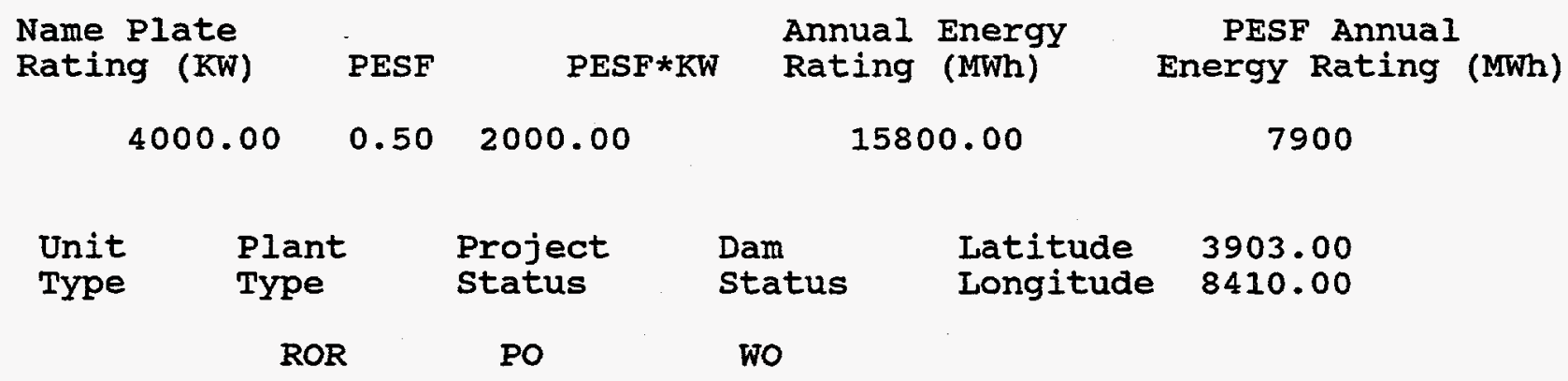

Factor

Exist Prob

Factor

Exist Prob

Wild/Scenic Protection

Wild/Scenic Tributary or Upstream/Downstream wild/Scenic Location Cultural Value Fish Presence Value Geologic Value Historic Value other Value Recreation Value Scenic Value

Exist Prob

$Y$
0.90 Wildlife Value Threatened/Endangered $\mathrm{Fish}$ Threatened/Endangered Wildlife

0.75 Federal Land Code 103

0.90 Federal Land Code 104

0.90 Federal Land Code 105

0.90 Federal Land Code 106

0.90 Federal Land Code 107

0.90 Federal Land Code 108

0.90 Federal Land Code 198 
DATE: $12 / 01 / 97$

PAGE NO: 34

FERC

Number

Plant Name

Stream

State

Name

OHOO1 FREMONT

SANDUSKY $R$

$\mathrm{OH}$

County Name

River Basin

SANDUSKY

CATTARAUGUS CREEK BASIN

Class

Owner Name

M FREMONT, CITY OF

Name Plate

PESF

Annual Energy

Rating (KW)

$0.10 \quad 470.00$

Rating (MWh)

PESF Annual

4700.00

PESF*KW

9850.00

985

$\begin{array}{cllll}\text { Unit } & \text { Plant } & \text { Project } & \text { Dam } & \text { Latitude } \\ \text { Type } & \text { Type } & \text { Status } & \text { Status } & \text { Longitude } \\ & \text { ROR } & & \text { U } & \end{array}$

Factor

Exist Prob

Factor

Exist Prob

wild/Scenic Protection

Wild/Scenic Tributary or

$\mathrm{Y} \quad 0.10$ Wildlife Value

Threatened/Endangered Fish
Threatened/Endangered Wild

4120.00

Upstream/Downstream wild/Scenic Location

Cultural value

Threatened/Endangered Fish
Threatened/Endangered wildlife

Fish Presence Value

Geologic Value

Historic Value

Other Value

Recreation Value

Scenic Value

0.90 Federal Land Code 103

0.90 Federal I and Code 104

0.90 Federal Land Code 105

0.90 Federal Iand Code 106

8308.00

$\begin{array}{cccc} & 0.90 & \text { Federal Land Code } 103 \\ & 0.90 & \text { Federal Land Code } 104 \\ & 0.90 & \text { Federal Land Code } 105 \\ & 0.90 & \text { Federal Iand Code } 106 \\ \text { Y } & 0.50 & \text { Federal Land Code } 107 \\ & 0.90 & \text { Federal Land Code } 108 \\ \text { Y } & 0.25 & \text { Federal Land Code } 198 \\ & 0.90 & \end{array}$

0.90

0.90

0.90

0.90

0.90

0.90

0.90

0.90

0.90

0.90 
DATE: $12 / 01 / 97$

FERC

Number

OHOO2 DAYTON LOW DAM

County Name

MONTGOMERY
PAGE NO: 35
Plant Name

\section{Class}

M

Owner Name

MIAMI CONSERVANCY DIST

Name Plate

Rating ( $\mathrm{KW}$ )

PESF

Annual Energy

$50.00 \quad 0.50 \quad 25.00$

PESF * KW

Rating (MWh)

144.00

State

Name

$\mathrm{OH}$

GREAT MIAMI $R$

River Basin

OHIO MAIN STREAM

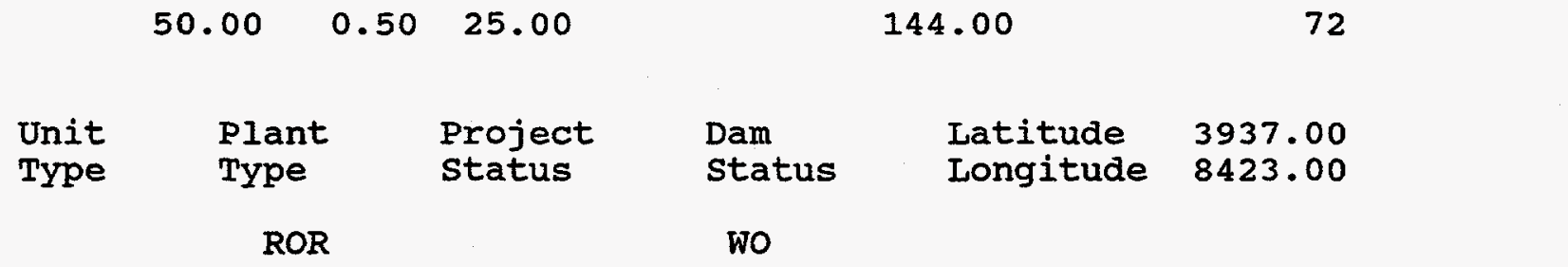

Factor

Wild/Scenic Protection wild/Scenic Tributary or Upstream/Downstream wild/Scenic Location Cultural value Fish Presence Value Geologic Value Historic Value other value Recreation value Scenic Value
Exist Prob

0.90

Wildife Value Threatened/Endangered Fish Threatened/Endangered Wildlife

Exist Prob

$\begin{array}{ll}0.90 & \text { Federal Land Code } 103 \\ 0.75 & \text { Federal Land Code } 104\end{array}$

Y $\quad 0.75$

Federal Land Code 105

0.90 Federal Land Code 106

$\mathrm{Y} \quad 0.75$

Federal Land Code 107

0.90 Federal Land Code 108

Y $\quad 0.75$ Federal Land Code 198
0.90

0.90

0.90

0.90

0.90

0.90

0.90

0.90

0.90

0.90
PESF Annual Energy Rating (MWh)

72 
DATE: $12 / 01 / 97$

PAGE NO: 36

FERC

Number

Plant Name

stream

St:ate

OH003 C J BROWN

BUCK CR, MAD R

Name

OHO03

county Name

River Basin

CLARK

OHIO MAIN STREAM

Class Owner Name

F CORPS OF ENGINEERS

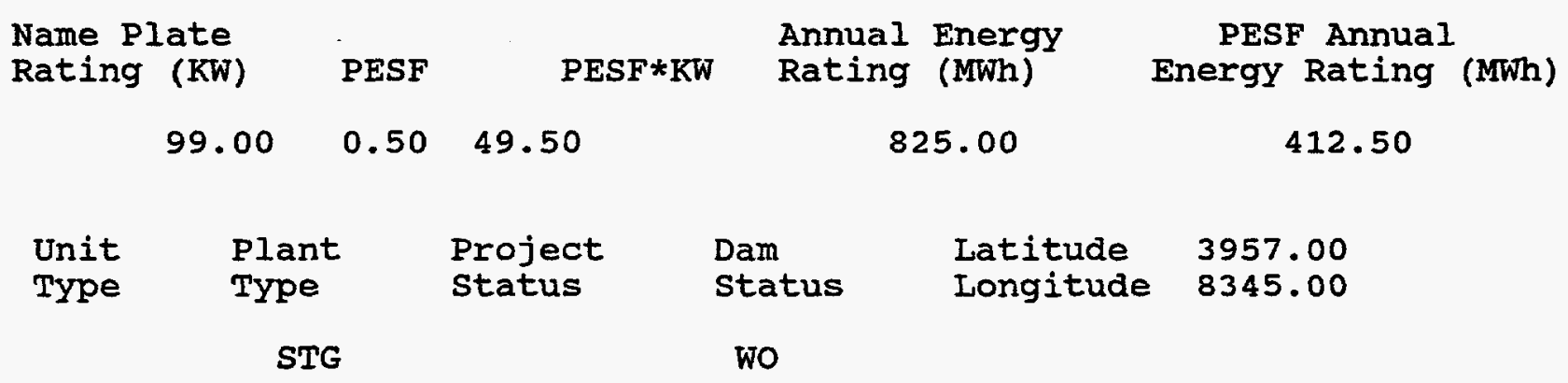

Factor

Exist Prob

Factor

Exist Prob

wild/Scenic Protection

wild/Scenic Tributary or

Upstream/Downstream wild/Scenic Location

Cultural value

Fish Presence Value

Geologic Value

Historic Value

other Value

Recreation Value

Scenic Value

Exist Pro

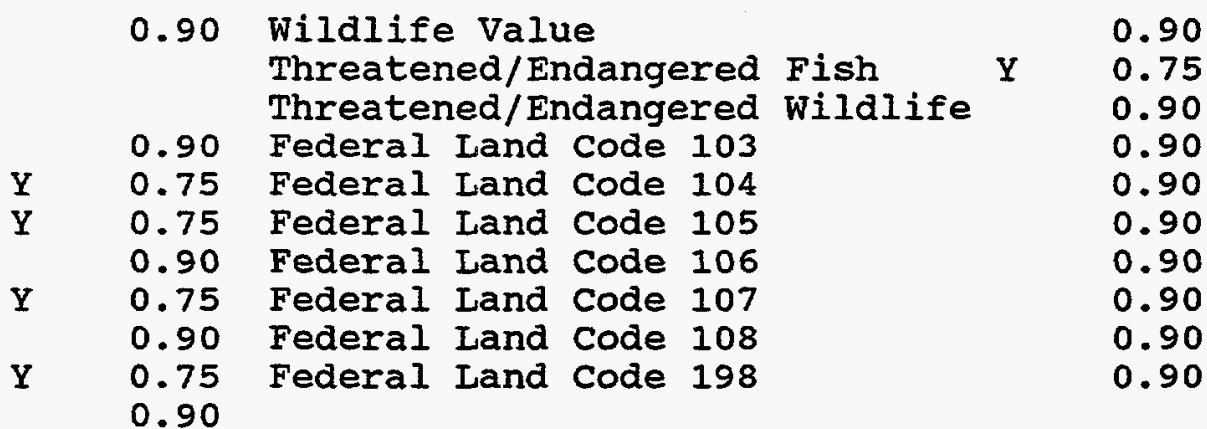


DATE: $12 / 01 / 97$

PAGE NO: 37

FERC

Number

Plant Name

stream

state

ISLAND PARK

GREAT MIAMI $R$

Name

$\mathrm{OHOO4}$

county Name

River Basin

MONTGOMERY

OHIO MAIN STREAM

class Owner Name

M DAYTON, CITY OF

Name Plate

PESF

Annual Energy

Rating ( $\mathrm{KW}$ )

PESF*KW

Rating (MWh)

PESF Annual

50.00

$0.50 \quad 25.00$

144.00

72

$\begin{array}{ccc}\text { Unit } & \text { Plant } & \begin{array}{l}\text { Project } \\ \text { Type }\end{array} \\ & \text { Type } & \text { Rotus } \\ & \end{array}$

$\begin{array}{lll}\text { Dam } & \text { Latitude } & 3955.00 \\ \text { Status } & \text { Longitude } & 8420.00 \\ \text { wo } & & \end{array}$

Factor

Exist Prob

Factor

Exist Prob

Wild/Scenic Protection

wild/Scenic Tributary or Upstream/Downstream wild/Scenic Location cultural value

Fish Presence Value

Geologic Value

Historic Value

other Value

Recreation Value

Scenic Value

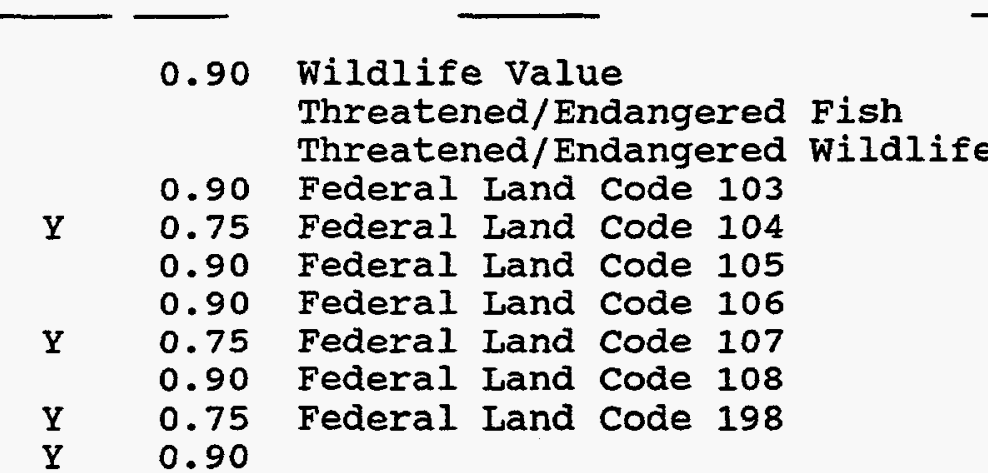

0.90

0.90

0.90

0.90

0.90

0.90

0.90

0.90

0.90

0.90 
R E S O U R C E D A T A B A S E I I S T I N G

DATE: $12 / 01 / 97$

PAGE NO: 38

FERC

Number

Plant Name

stream

State

Name

OHOO5 PIQUA

MIAMI $\mathbf{R}$

$\mathrm{OH}$

County Name

SHELBY
River Basin

OHIO MAIN STREAM

Class Owner Name

M PIQUA MUNICIPAL PWR SYS

Name Plate

PESF

Annual Energy

PESF*KW

PESF Annual

Rating (KW)

$0.90 \quad 720.00$

Unit Type

Plant
Type

ROR

Factor

Exist Prob

0.90

Wild/Scenic Protection

wild/Scenic Tributary or

Upstream/Downstream

wild/Scenic Location

Cultural value

Fish Presence Value

Geologic Value

Historic Value

other Value

Recreation Value

Scenic Value

\section{Project status}

Existe

Factor

Latitude

4021.00

Dam

status

Longitude

8416.00

U
Rating (MWh)

3000.00

2700

Energy Rating (MWh)

\begin{tabular}{lll}
\multicolumn{1}{c}{ Factor } & Exist Prob \\
& & \\
Wildlife Value & & 0.90 \\
Threatened/Endangered Fish & 0.90 \\
Threatened/Endangered Wildlife & 0.90 \\
Federal Land Code 103 & 0.90 \\
Federal Land Code 104 & 0.90 \\
Federal Land Code 105 & 0.90 \\
Federal Land Code 106 & 0.90 \\
Federal Land Code 107 & 0.90 \\
Federal Land Code 108 & 0.90 \\
Federal Land Code 198 & 0.90
\end{tabular}


RESOURCE

DATE: $12 / 01 / 97$
D A T A B A S E

(2)

I $T$ IN

PAGE NO: 39

FERC

Number

Plant Name

OHO06 RACINE L\&D

County Name

MEIGS

stream

State

Name

OHIO R

$\mathrm{OH}$

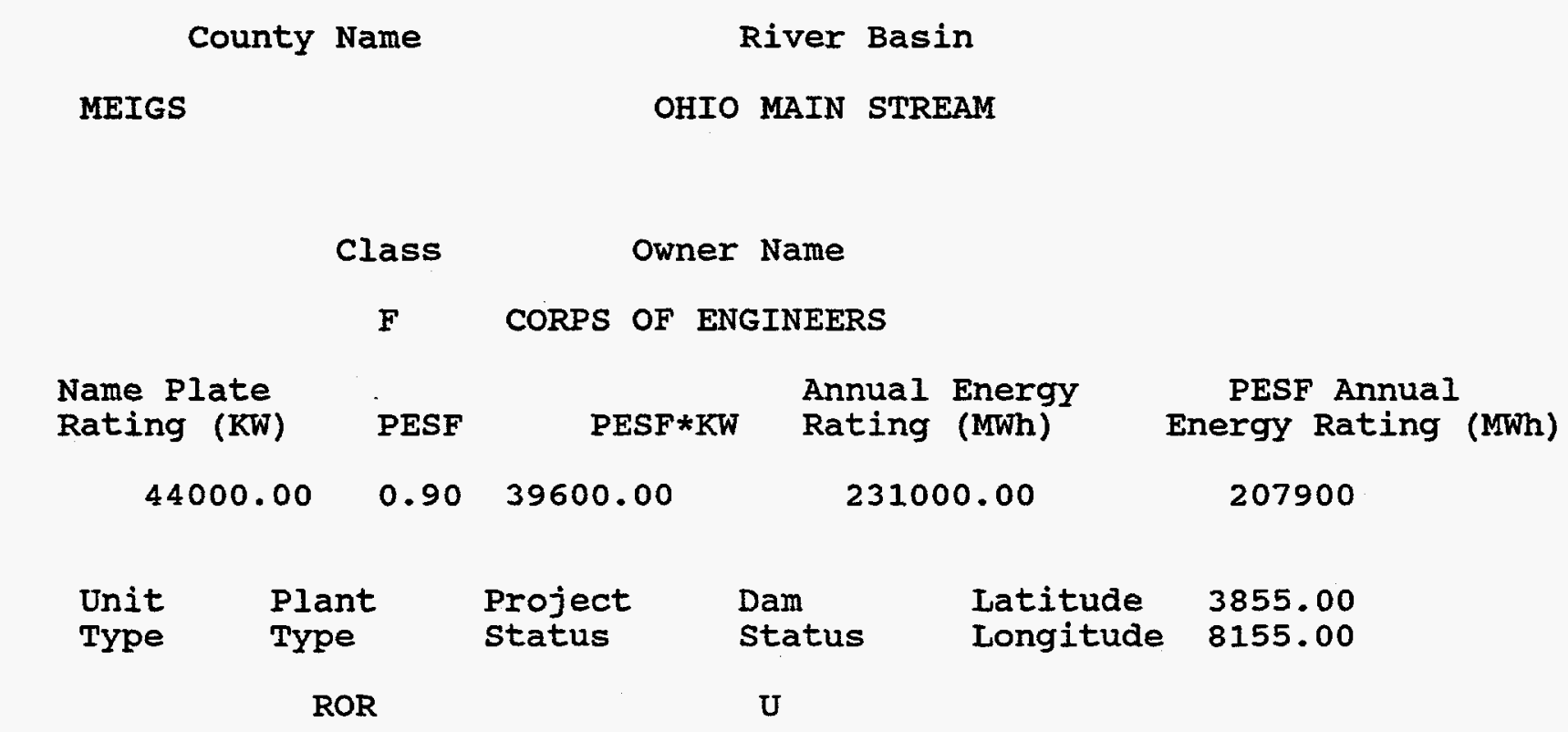

\author{
Factor \\ wild/scenic Tributary or \\ Upstream/Downstream \\ wild/Scenic Location \\ Fish Presence Value \\ Geologic Value \\ Historic Value \\ other value \\ Recreation Value
}

Exist Prob

Factor

Exist Prob

0.90

Wildlife value

Threatened/Endangered Fish

Threatened/Endangered Wildlife

0.90 Federal Land code 103

0.90 Federal Land Code 104

0.90 Federal Land Code 105

0.90

Federal Land Code 105
Federal Land Code 106

0.90 Federal Land Code 107

0.90 Federal Land Code 108

0.90 Federal Land Code 198

0.90

0.90

0.90

0.90

0.90

0.90

0.90

0.90

0.90

0.90

0.90 
DATE: $12 / 01 / 97$

FERC

Number

OH0O7 DOVER

County Name

TUSCARAWAS

Class

Owner Name

F CORPS OF ENGINEERS

Name Plate Rating (KW)

121.00
Annual Energy PESF*KW Rating (MWh)

1050.00

Latitude

Longitude

4033.00

Type

$\begin{array}{ll}\text { Plant } & \text { Project } \\ \text { Type } & \text { Status }\end{array}$

STG

Factor

Exist Prob

Factor

Exist Prob

wild/Scenic Protection

wild/Scenic Tributary or Upstream/Downstream wild/Scenic Location Cultural Value

Fish Presence Value

Geologic Value

Historic Value

other Value

Recreation Value

scenic Value

\section{Status}

wo
PESF Annual Energy Rating (MWh)
525

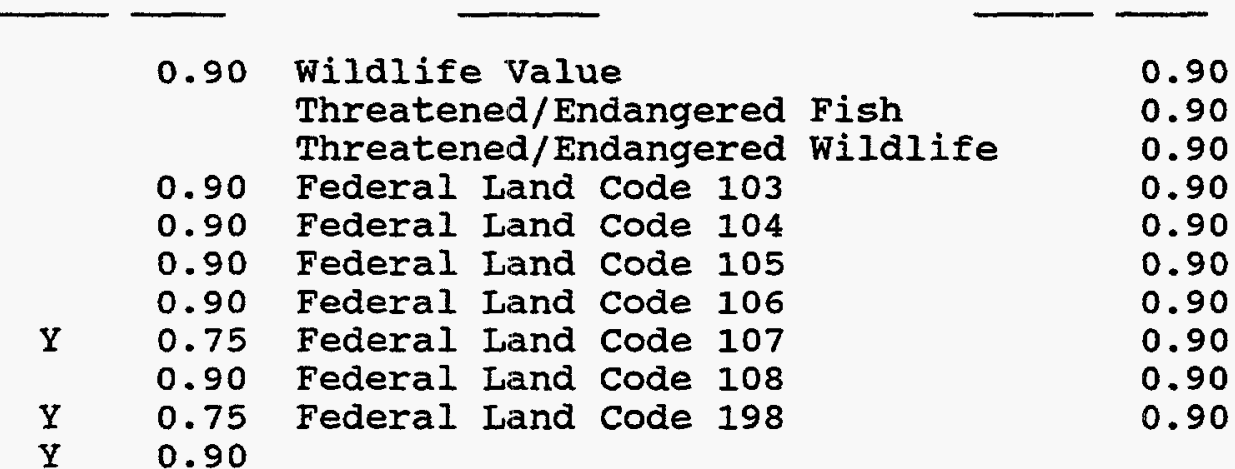

St:ate

Name

$\mathrm{OH}$ 
DATE: $12 / 01 / 97$

FERC

Number

Plant Name

ОН008

MOSQUITO CREEK

County Name

TRUMBULLL

class

Owner Name

F CORPS OF ENGINEERS

Name Plate

Rating (KW)

PESF

Annual Energy

1100.00

$0.90 \quad 990.00$

PESF*KW

Rating (MWh)

PAGE NO: 41
River Basin

BEAVER RIVER BASIN
State

Name

$\mathrm{OH}$

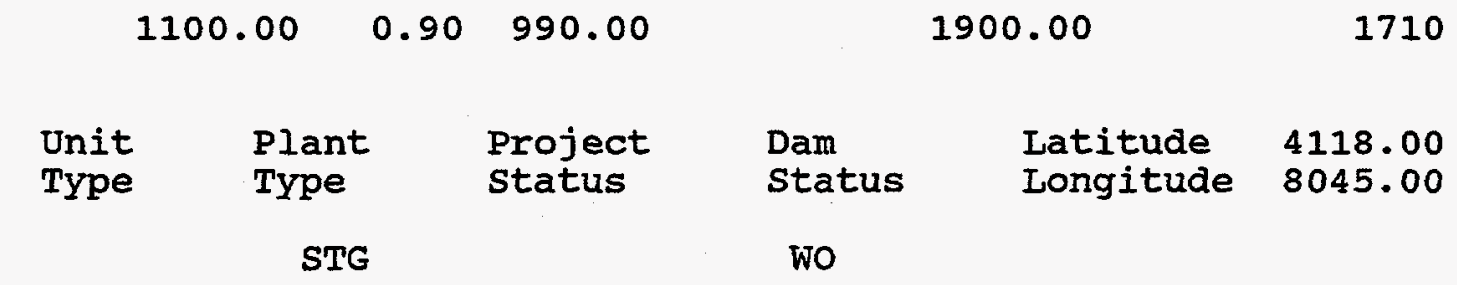

Factor

Exist Prob

Factor

Exist Prob

0.90

Wildiffe Value

0.90 Threatened/Endangered Fish

0.90

Wild/Scenic Tributary or Threatened/Endangered Wildlife

0.90

0.90 Federal Land Code 103

0.90 Federal Land Code 104

0.90

0.90 Federal Land Code 105

0.90

0.90 Federal Land Code 106

0.90

0.90 Federal Land Code 107

0.90

Geologic Value

Historic Value

0.90

Federal Land Code 108

0.90

0.90 Federal Land Code 198

0.90

Recreation value

0.90

0.90 
DATE: $12 / 01 / 97$

PAGE NO: 42

FERC

Number

Plant Name

Stream

State

OH009 MICHAEL J KIRWIN

County Name

River Basin

PORTAGE

BEAVER RIVER BASIN

$\begin{array}{cc}\text { Class } & \text { Owner Name } \\ \text { F } & \text { CORPS OF ENGINEERS }\end{array}$

Name Plate

Rating (KW)

PESF

Annual Energy

PESF*KW

Rating (MWh)

PESF Annual

1470.00

$0.90 \quad 1323.00$

4000.00

3600

$\begin{array}{cccccc}\text { Unit } & \text { Plant } & \text { Project } & \text { Dam } & \text { Latitude } & 4110.00 \\ \text { Type } & \text { Type } & \text { Status } & \text { Status } & \text { Longitude } & \mathbf{8 1 0 5 . 0 0} \\ & \text { STG } & & \text { wo } & \end{array}$

Factor

Exist Prob

Factor

Exist Prob

Wild/Scenic Protection

Wild/Scenic Tributary or

Upstream/Downstream

wild/scenic Location

Cultural value

Fish Presence Value

Geologic Value

Exist Prob

0.90 Wildife Value

Threatened/Endangered Fish

Threatened/Endangered Wildlife

$\mathrm{CH}$

Name

W BR MAHONING $R$

Historic Value

$\begin{array}{lll}0.90 & \text { Federal Land Code } 103 \\ 0.90 & \text { Federal Land Code } 104\end{array}$

$\begin{array}{ll}0.90 & \text { Federal Land Code } 104 \\ 0.90 & \text { Federal Land code } 105\end{array}$

0.90 Federal Land Code 106

0.90 Federal Land Code 107

other value

Recreation value

0.90 Federal Land code 108

0.90 Federal Land Code 198

Exist Prob

0.90

0.90

0.90

0.90

0.90

0.90

0.90

0.90

0.90

0.90

Scenic Value

0.90 

R E S O U R C E
D A T A B A S E
L I S T I N G

DATE: $12 / 01 / 97$

PAGE NO: 43

FERC

Number

Plant Name

stream

state

OHO10 IAKE MILTON

MAHONING $\mathrm{R}$

Name

County Name

River Basin

MAHONING

BEAVER RIVER BASIN

OH

\author{
Class Owner Name \\ M YOUNGSTOWN, CITY OF
}

Name Plate

Rating (KW)

Annual Energy

$2570.00 \quad 0.90 \quad 2313.00$

PESF

Rating (MWh)

PESF Annual

Energy Rating (MWh)

5610.00

5049

$\begin{array}{clllll}\text { Unit } & \text { Plant } & \text { Project } & \text { Dam } & \text { Latitude } & 4106.00 \\ \text { Type } & \text { Type } & \text { Status } & \text { Status } & \text { Longitude } 8059.00 \\ & \text { STG } & & \mathrm{U} & \end{array}$

Factor

Exist Prob

Factor

Exist Prob

Wild/Scenic Protection

wild/Scenic Tributary or

0.90 Wildlife Value

Threatened/Endangered $\mathrm{Fish}$

Upstream/Downstream

Threatened/Endangered Wildlife

0.90

0.90

wild/scenic Location

0.90 Federal Land Code 103

0.90

0.90 Federal Land Code 104

0.90

Cultural value

0.90 Federal Land Code 105

0.90

0.90 Federal Land Code 106

0.90

Geologic Value

0.90 Federal Land Code 107

0.90

Historic Value

0.90

Federal Land Code 108

0.90

Other Value

0.90 Federal Land Code 198

0.90

Scenic Value

0.90

0.90 